\title{
1 Single-cell Stereo-seq enables cell type-specific spatial 2 transcriptome characterization in Arabidopsis leaves
}

\section{Authors:}

5 Keke Xia ${ }^{1, \#}$, Hai-Xi Sun ${ }^{1, \#}$, Jie Li ${ }^{1,2, \#}$, Jiming Li ${ }^{1, \#}$, Yu Zhao ${ }^{1, \#}$, Ruiying Chen ${ }^{1,3}$, Guangyu Liu ${ }^{1}$, 6 Zhiyong Chen ${ }^{1}$, Ruilian Yin ${ }^{1,2}$, Shijie $\mathrm{Hao}^{1,2}$, Jing Wang ${ }^{1}$, Qing Xie ${ }^{1}$, Jiangshan $\mathrm{Xu}^{1,2}$, 7 Yuxiang $\mathrm{Li}^{1}$, Ao Chen${ }^{1}$, Longqi Liu ${ }^{1,4}$, Ye Yin ${ }^{5}$, Huanming Yang ${ }^{1,6}$, Jian Wang ${ }^{1,6, *}$, Ying $8 \mathrm{Gu}^{1,7, *}$ and $\mathrm{Xun} X \mathrm{u}^{1,7, *}$

$10{ }^{1}$ BGI-Shenzhen, Shenzhen 518083, Guangdong, China

112 College of Life Sciences, University of Chinese Academy of Sciences, Beijing 100049,

12 China

$13{ }^{3}$ College of Informatics, Huazhong Agricultural University, Wuhan 430070, Hubei, China

$14{ }^{4}$ Shenzhen Key Laboratory of Single-Cell Omics, BGI-Shenzhen, Shenzhen 518120,

15 Guangdong, China

$16{ }^{5}$ BGI Genomics, BGI-Shenzhen, Shenzhen 518083, Guangdong, China

$17{ }^{6}$ James D. Watson Institute of Genome Sciences, Hangzhou 310058, Zhejiang, China.

187 Guangdong Provincial Key Laboratory of Genome Read and Write, BGI-Shenzhen,

19 Shenzhen 518120, Guangdong, China

20 \# These authors contributed equally to this work. 


\section{Summary}

27 Understanding the complex functions of plant leaves requires spatially resolved gene expression profiling with single-cell resolution. However, although in situ gene expression

29 profiling technologies have been developed, this goal has not yet been achieved. Here, 30 we present the first in situ single-cell transcriptome profiling in plant, scStereo-seq (single31 cell SpaTial Enhanced REsolution Omics-sequencing), which enabled the bona fide 32 single-cell spatial transcriptome of Arabidopsis leaves. We successfully characterized 33 subtle but significant transcriptomic differences between upper and lower epidermal cells.

34 Furthermore, with high-resolution location information, we discovered the cell type-specific spatial gene expression gradients from main vein to leaf edge. By reconstructing those

36 spatial gradients, we show for the first time the distinct spatial developmental trajectories

37 of vascular cells and guard cells. Our findings show the importance of incorporating spatial 38 information for answering complex biological questions in plant, and scStereo-seq offers a powerful single cell spatially resolved transcriptomic strategy for plant biology.

\section{Keywords:}

43 Single-cell Stereo-seq, Single-cell spatial transcriptome, spatial developmental trajectory, 44 leaves, Arabidopsis 


\section{INTRODUCTION}

48 Leaves are one of the most important organs for plants. In fully grown leaves, the main leaf cell types include epidermis, mesophyll and vasculature. In Arabidopsis, the epidermis

50 is composed of guard cells and trichomes that are embedded in the single cell layer of

51 pavement cells. The upper and lower epidermal cells enclose the palisade and spongy mesophyll, which represent the main photosynthetic capacity of the leaf. Embedded in the mesophyll cells are the vascular cells(Svozil et al., 2015; Tsukaya, 2013). Since individual cell types have specialized functions during leaf development and response to environmental stimuli, a comprehensive in situ cell type-specific multi-omics profiling is needed for understanding the complexity of plant leaves. However, this has not yet been

\section{7 achieved.}

Recently, high-throughput single cell RNA sequencing (scRNA-seq) technology provides new insights into leaf cell biology, which has facilitated our understanding of stomata and vasculature development at single-cell level(Kim et al., 2021; Liu et al., 2020b). Despite its advantage in single-cell resolution, its application to plant leaf studies still faces challenges(Gurazada et al., 2021). First, protoplasting is still difficult for many plants leaf and other organs, and it also causes changes in the expression of hundreds of genes, which may influence the following transcriptome profiling(Tian et al., 2020). Second, many cell types are resistant to protoplasting, and protoplasting may yield bias proportions of cell types(Bezrutczyk et al., 2021). And third, the most important, the tissue dissociation leads to the loss of spatial information of cells. Spatial information is not only important for the analysing of cell-cell interaction and cell-environment interaction but also crucial for cell type identification, especially for cell types with limited knowledge on marker genes. With spatial information, it could be possible to distinguish cell sub-types and analyze

72 subtle transcriptomic differences between cell sub-types, such as between upper and

73 lower epidermal cells and between palisade and spongy mesophyll cells(Kim et al., 2021; 
74 Liu et al., 2020b). Since these drawbacks of scRNA-seq for plant studies, the development

75 of in situ gene expression profiling technologies are motivated.

A number of remarkable high-throughput transcriptomic profiling of in situ gene expression methodologies have been developed recently(Chen A. et al., 2021; Chen et al., 2015; Cho et al., 2021; Eng et al., 2019; Jiao et al., 2009; Lee et al., 2014; Liu et al., 2020a; Nichterwitz et al., 2016; Rodriques et al., 2019; Srivatsan et al., 2021; Stahl et al., 2016; Stickels et al., 2021; Vickovic et al., 2019; Wang et al., 2018), such as Slide-seq, DBiTseq (deterministic barcoding in tissue for spatial omics sequencing). Up to now, most of these methods only demonstrate their applications in mammalian systems, and only one method has been applied in plant systems so far (Giacomello et al., 2017). However, the achieved resolution of this approache is still at tissue/domain level, rather than single-cell level. Among all in situ gene expression methodologies, Stereo-seq (SpaTial Enhanced REsolution Omics-sequencing) is so far the only sequencing-based spatially resolved transcriptomic technology with subcellular resolution(Chen A. et al., 2021). This technique exhibits a great potential for achieving single cell resolution in situ gene expression profiling in plant biology. resolution spatial transcriptomics using Stereo-seq, we present the first in situ single-cell transcriptome profiling in Arabidopsis leaves. Single-cell Stereo-seq (scStereo-seq) not only enables us to identify the main leaf cell types, but also allows us to distinguish cell sub-types, including upper and lower epidermal cell, palisade mesophyll cell and spongy mesophyll cell. Furthermore, we also show the existence of cell type-specific spatial gene expression gradients from main vein to leaf edge. We reconstructed those gradients to show for the first time the developmental trajectories of specific cell types according to 
101 their spatial distribution.

\section{RESULTS}

\section{Establishing single-cell Stereo-seq method in Arabidopsis leaves}

105 To obtain in situ transcriptome profiling at single-cell level, we set out to apply Stereo-seq 106 in Arabidopsis leaves. We employed eleven Arabidopsis cauline leaf cross sections to explore the applicability of Stereo-seq in the plant system (Figure 1A). A. thaliana cauline leaves were cryosectioned and positioned on top of the chip surface with DNA nanoball (DNB) docked in a grid-patterned array of spots, each spot with a size of $220 \mathrm{~nm}$ in diameter and a center-to-center distance of $715 \mathrm{~nm}$. The DNB contains random barcoded sequences, the coordinate identity (CID), molecular identifiers (MID) and polyT sequencecontaining oligonucleotides, and the polyT was designed to capture mRNAs. After cell wall staining and imaging, the same section was sequenced with Stereo-seq. Finally, eleven leaves with relatively good morphology from two chips were selected for further analysis.

115 To assess whether the detected transcript signal was stringently confined in leaf areas in 116 the bright field, we assessed the molecular identifiers (MID) distribution. Clearly, the MID 117 distribution was specially localized in the leaf areas, and no transcript signal diffusion was observed (Figure 1B, S1A and S1B).

120 To visualize cell-cell boundary, we performed toluidine blue (TB) staining of plant cell wall on the same section we used for Stereo-seq. After merging the staining image and sequencing data in the Stereomics visualization system, we then used lasso tool to

123 manually extract single-cells based on cell wall boundary and coordinated them with MID 124 counts to obtain specific data for each single cell. For eleven leaf samples, we obtained 1,177 cells in total, and these cells had clear cell boundaries and could be clearly assigned to specific cell types. After filtering (gene number: 200-5000; percentage of mitochondrial

127 gene: < $10 \%$; see Methods), a total of 871 high-quality single cells were subject to further 
analyses. We then determined the gene number, the MID number, the percentage of mitochondria genes, and the percentage of chloroplast genes in each cell to evaluate our data quality. The majority cells had similar gene numbers and MID numbers with a strong positive correlation (Figure $1 \mathrm{C}$ and $\mathrm{S1C}$ ), and there was no obvious difference among the eleven leaves (Figure 1D), providing clear evidence of consistency and reproducibility. In addition, when we compared the data obtained by single-cell method mentioned above with that obtained by the standard Stereo-seq data analysis method (the binning method using 20 x 20 DNBs, hereafter referred to as 'Bin20') (Chen A. et al., 2021), we observed a significant increase in gene and MID numbers of the single-cell method, indicating that single cell extraction greatly improved the data quality of Stereo-seq (Figure 1C). Taken together, we obtained spatially resolved single-cell transcriptome profiling of Arabidopsis cauline leaves.

\section{Obtaining cell type-specific transcriptomes at bona fide single-cell level}

With morphology features and their locations in the leaves, the 871 cells were classified into 226 epidermal cells (130 upper and 96 lower epidermal cells), 472 mesophyll cells (331 palisade and 141 spongy mesophyll cells), 95 guard cells and 78 vascular cells (Figure 2A and Table S1). The gene number, UMI number, percentage of mitochondria genes and the percentage of chloroplast genes per cell showed similar patterns in four different major cell types (Figure 2B), demonstrating that our approach was able to obtain unbiased single-cell transcriptome of all major cell types of Arabidopsis leaves.

In order to confirm the reliability of cell type-specific transcriptome, we performed the marker genes, Principle Component Analysis (PCA), and differentially expressed genes (DEGs) analyses among the four major cell types. All four major cell types expressed celltype-specific marker genes (top five up-regulated genes were listed) (Figure 2C). Meanwhile, the percentage of cells expressing cell-type markers of single-cell method was 
significantly higher than those of Bin20 (Figure 2D and S2A-D), showing that the singlecell method could better reflect the cell-type-specific transcriptome characteristics. As a result, PCA and differential expression analyses using the single-cell transcriptome revealed that the same cell type from different leaves was clustered together while different cell types from different leaves were separately grouped (Figure 2E and S2E-I). These significant differences among the four major cell types demonstrated the consistency and reproducibility of scStereo-seq data at cell-type level.

We further validated the reliability of the four cell types we distinguished in two aspects. On the one hand, we detected the expression level of the previously reported marker genes in each cell type, GSTU20, AT2G45860, AT5G53940, and HSP81-2, which were specifically expressed in vascular cells, epidermal cells, mesophyll cells and guard cells, respectively(Kim et al., 2021; Liu et al., 2020b; Zhang et al., 2021). And the results showed that these genes were highly expressed in their corresponding cell types and lowly expressed in other cell types (Figure 3A and S3A). In addition, these marker genes were specifically expressed in their corresponding loci in the TB staining images (Figure 3A and S3A). On the other hand, we investigated the biological processes enriched in each cell type, and found that all cell types were enriched in biological processes corresponding to their specialized functions (Figure 3B). For instance, vascular cells were enriched in transportation-related pathways ${ }^{38,39}$, while mesophyll cells were enriched in photosynthesis-related pathways and sucrose metabolic process(Ruan, 2014). Three photosynthesis-related Gene Ontology (GO) terms were also found in vascular cells, and this feature has been experimentally validated in the vascular tissues of phylogenetically widespread C3 plants(Brown et al., 2010; Gao et al., 2018; Hibberd and Quick, 2002). These results demonstrated the reliability of cell type identification using extracted singlecell data using scStereo-seq. So far, we obtained in situ reliable cell type-specific transcriptome data at single-cell level, for the first time. 
183 Spatial information and cell boundary are necessary to identify subtle 184 transcriptional differences between cell sub-types

185 For Arabidopsis leaves, epidermis can be subdivided into upper epidermal cell and lower 186 epidermal cell, and mesophyll cell can also be subdivided into spongy mesophyll cell and 187 palisade mesophyll cell. However, because of highly similar transcriptomes between cell 188 sub-types, scRNA-seq has not been able to distinguish these cell sub-types in previous 189 studies(Kim et al., 2021; Liu et al., 2020b). In this study, using scStereo-seq, we could 190 extract single cells and classify cell types based on TB staining of cell walls and spatial 191 histological information. Therefore, we were able to distinguish upper and lower epidermal 192 cells, as well as spongy and palisade mesophyll cells: Without the spatial histological 193 information, cell sub-types could not be distinguished using single cell transcriptome alone 194 (Figure 3C and S3B); without the accurate cell boundaries (the Bin20 method), they could 195 not be distinguished either (Figure S3E and S3F). Because inaccurate cell boundaries 196 conferred poor data quality (Figure 1C) and fewer transcriptomic features (Figure 2D), we 197 demonstrated that both spatial coordinate and accurate cell boundaries were required for 198 cell sub-type classification in plant leaves, and also showed the necessity of using scStereo-seq technology for complex plant tissue studies.

With obtained datasets and DEGs of these cell sub-types, we further investigated their functional differences, which has significant biological implications and has not yet been achieved so far. As shown in Figure 3E, biological processes associated with high light response, wax biosynthetic process and response to insect were enriched in upper epidermal cell, while biological terms related to freezing, membrane lipid metabolic process and regulation of programmed cell death, were significantly enriched in lower epidermal cell (Figure 3E). For instance, ERD7 is upregulated in various biological 
209

210

211

212

213

214

215

216

217

218

219

220

221

222

223

224

225

226

227

228

229

230

231

232

233

234

235

induced lipocalin (TIL) is involved in cold stress response(Frenette Charron et al., 2002;

Kawamura and Uemura, 2003; Miki et al., 2019). In this study, using scStereo-seq, we further discovered that ERD7 and TIL were highly expressed in upper epidermal cell and lower epidermal cell, respectively (Figure 3D), indicating upper and lower epidermal cells probably were functionally different in response to high light and cold stress. For mesophyll cell sub-types, we also found that oxygen-related processes were enriched in spongy mesophyll cells (Figure S3D). Taken together, by distinguishing cell sub-types, we revealed the subtle transcriptional differences between upper and lower epidermal cells, spongy and palisade mesophyll cells, which would enable us to discover the complex regulation mechanisms during leaf development and in response to environmental stimuli, in future studies.

\section{Photosynthesis-related genes exhibited expression gradient from main vein to leaf} edge

Genes specifically expressed in certain areas of leaves play key roles in various plant biological processes, such as leaf development and plant photomorphogenesis(Martinez et al., 2021; Tian et al., 2019; Wang et al., 2020). As spatial information of individual cells was documented by scStereo-seq, we were able to assess gene expression levels in different parts of leaves at single cell level, for the first time. As shown in Figure 4A, the main vein area was designated as Area0, and the Area on the one side of the main vein was divided into 3 areas, which were designated as Area 1, 2 and 3, and Areas 1 to 3 gradually away from the main vein. We then determined gene expression patterns in the designated areas of the leaves.

Interestingly, 3 patterns of spatial gene expression gradients from main vein to leaf edge were observed when we performed gene expression pattern analysis along the leaf for all cells together (Figure 4B). Pattern 1 genes were highly expressed in the main vein (Area 
0) and had a low, relatively stable expression in Area 1, 2 and 3. Pattern 2 genes were highly expressed in Area 1, and the expression level of Pattern 3 genes increased gradually from Area 0 to 3 . We found that photosynthesis-related biological processes, such as photosynthesis, photosynthesis electron transport chain and photosynthesis (light reaction), were highly enriched in Pattern 2 (Figure 4C), suggesting that the photosynthetic efficiency decreases gradually from the middle to the edge of the leaf. Given that the photosynthesis process mainly takes place in mesophyll cells and to further validate this observation, we performed the same analysis on mesophyll cell. Three gene expression patterns similar to those of all cells were observed (Figure 4E), and consistently, the photosynthesis-related biological processes were also highly enriched in Pattern 2 in mesophyll cells (Figure 4F). Representative genes were presented, including PQL1, LHCA6, PSB29, PPL2 and FNR1 (Figure 4D and 4G), which are known regulators of photosynthesis process(Ishihara et al., 2007; Keren et al., 2005; Morales et al., 2000; Peng et al., 2009; Yabuta et al., 2010). These results further supported that photosynthesis process exhibited a spatial distribution in plant leaves and the photosynthetic efficiency might decrease gradually from the middle to the edge of the leaf. The elevated expression levels of photosynthesis-related genes in Area 1 indicated that photosynthesis was involved in a sophisticated and complex regulation mechanism in leaves, and future studies are needed to uncover the spatial gene regulatory mechanisms.

\section{Vascular cells and guard cells exhibit distinct spatially resolved developmental} trajectories

It isknown that the differentiation at the margin of a leaf is decelerated relative to the more medial regions(Martinez et al., 2021). However, it is still unknown whether all cell types in leaves follow the same development pattern. Here, to address these questions and taking advantage of spatial information of scStereo-seq, we performed pseudotime analysis on vascular cell and guard cell, and compared their developmental stages in the 4 areas as 
described in last section (Figure 4A).

265 Pseudotime analysis on vascular cells showed one major trajectory with vascular cells 266 from different leaf areas separately located along the pseudotime path (Figure 5A).

267 Vascular cells from Area 3, the relatively young area in leaves, were distributed at a 268 relatively early pseudotime stage, while vascular cells from Area 2, 1 and 0 were 269 distributed to the end of pseudotime branch (Figure 5A), suggesting that the vascular cells 270 from Area 3 were newly formed relative to the cells from Area 2, 1, and 0 . These results 271 showed that the development of vascular cells at the margin of a leaf is decelerated 272 relative to the more medial regions.

274 Moreover, to further investigate the functions of cells in different developmental stages, we 275 assessed gene expression pattern of DEGs along the pseudotime (Figure 5B). DEGs were 276 classified into three distinct clusters. Cluster 1 genes were highly expressed genes at the 277 late stage of the pseudotime, corresponding to cells in the main vein area, and their 278 functions were mainly involved in toxin-related biological processes via GO. Cluster 2 279 genes that were mainly upregulated in the middle stage of pseudotime were related to 280 photosynthesis, indicating that the photosynthesis process may play significant roles in 281 the development of cells from Area 1 or 2. For cluster 3, responses to oxygen were 282 enriched at the early stage of the pseudotime, corresponding to the leaf edge. Genes 283 involved in vascular development have been studied extensively before(Ischebeck et al., 284 2013), but their spatial-temporal expression patterns have not been clearly determined. 285 Thus, we determined the pseudotime expression profiles for representative genes from 286 different clusters (Figure 5C). GSTU20, AT5G44580 and AT1G09932 are probably 287 involved in lignin biosynthesis(Geng et al., 2019). GSTU20 mainly expressed in Area 0, 288 corresponding to the early formed main vein, while AT1G09932 mainly expressed in Area 2893 , corresponding to the latest formed cells, and AT5G44580 mainly expressed in cells form 
Area 1 and 2. And several other genes involved in vasculature development also showed spatial-temporal expression changes along the pseudotime (Figure S4A). These results indicated that the maturation of vascular cells went through several development stages, and complex spatial-temporal gene expression regulation was involved in these development stages. In future studies, to better reveal the complex regulatory network of vascular development, it is necessary to conduct independent studies at various development stages.

Because epidermal cell and guard cell differentiate from the same basal epidermal cell(Glover et al., 2016), we investigated the spatial developmental trajectory of epidermal cell and guard cell together. As shown in Figure 5D, epidermal cells and guard cells are located on different branches with one branch mainly containing epidermal cells, designated as cell fate 1 , and another branch, designated as cell fate 2, predominantly containing guard cells. More than $80 \%$ cells on cell fate 2 were guard cells, which was significantly higher than those on pre-branch and cell fate 1 (Figure 5E). However, when adding spatial information to the development trajectory, we did not find a clear spatial distribution pattern for cells from pre-branch and fate 1 and 2: no obvious difference in spatial distribution of these cells from Area 0 to 3 (Figure S4B and S4C). These data suggest that the development of vascular cells, but not guard cells, at the margin of leaf is decelerated relative to the more medial regions. The development stages with spatial information revealed here could provide important references for future studies on special development stages of vascular cells.

\section{DISCUSSION}

Leaves are the main photosynthetic organs, and they are also involved in responses to biotic and abiotic stresses(Maugarny-Cales and Laufs, 2018). Different cell types in plant leaves exhibit various molecular signatures and perform specific functions. Cell type- 
317 specific characterizations are essential for better understanding of the developmental 318 mechanisms of leaves and their responses to environmental stimuli. With the advantages of scStereo-seq that was developed in this study, we successfully revealed the complex cell type-specific and spatial-temporal gene expression features of Arabidopsis leaves.

Though plant cell wall is considered to bring difficulties in cell isolation for most plant single-cell studies, our study innovatively uses it for accurate single cell extraction by combining plant cell wall staining with Stereo-seq to obtain well-displayed cell-cell boundaries. The single-cell extraction method displayed a great improvement in data quality when compared with the Standard Stereo-seq Bin20 method. This enables us to generate the first bona fide single-cell spatial transcriptome profile in plants and to clearly distinguish cell types and cell sub-types. Although laser capture microdissection(Martinez et al., 2021), MERFISH (multiplexed error-robust FISH)(Xia et al., 2019) and 1cell-DGE (single cell-digital gene expression)(Kubo et al., 2019) can also perform single cell-types and single cell profiling with spatial information in plants, these methods suffer from low throughput and require special equipment which cannot be easily accessed in regular labs. In contrast, transcriptome signals of cells from eleven leaf sections were easily captured on chips in one time using our method, and the high-throughput in situ single cell transcriptome profile method allowed us to conveniently capture cells in batch and without the need of special equipment. Although the classification of epidermal cell, mesophyll cell, vascular cell, and guard cell types have been achieved using single cell transcriptomes by scRNA-seq in previous studies(Liu et al., 2020b; Lopez-Anido et al., 2021), identification cell sub-types such as upper and lower epidermal cell, and spongy and palisade mesophyll cell, have not been well achieved so far. In this study, we demonstrate that using single

342 sub-types, most likely because these cell sub-types have highly similar transcriptome 343 characteristics and the lacking of cell type-specific marker genes. In contrast, combining 
spatial information with single cell transcriptomes, we successfully identified these cell sub-types and obtained the cell type-specific characterizations. Thus, scStereo-seq can be easily extended to a variety of model or non-model plant species, avoiding the need of well-established marker genes for highly similar cell types identification.

Furthermore, using scStereo-seq, bona fide single-cell transcriptome data could be obtained without preparing protoplasts, thus avoiding the effects on gene expression and biased cell capture(Rich-Griffin et al., 2020; Shaw et al., 2021). The obtained dataset may better reflect the real transcriptional characteristics of the cell types in leaves. For highthroughput single-cell transcriptome analysis in Arabidopsis leaves, a large number of single protoplasting cells are needed for capturing rare cell types(Kim et al., 2021; Liu et al., 2020b; Lopez-Anido et al., 2021). And for scRNA-seq, optimized methods might be needed for capturing special cell types(Kim et al., 2021). These problems can be avoided by using scStereo-seq technology.

Based on cell spatial information, we found the existence of cell type-specific spatial gene expression gradients from main vein to leaf edge, such as FNR1, PSB29, and LHCA6 in mesophyll cells, which are important components of photosynthesis(Ishihara et al., 2007; Keren et al., 2005; Morales et al., 2000; Peng et al., 2009; Yabuta et al., 2010). The extremely low expression of photosynthesis-related genes in the main vein region (Area 0 ) is consistent with the main function of leaf vasculature, which plays a key role in solute translocation rather than in photosynthesis, reflecting the reliability of the spatial gene expression pattern. The expression levels of FNR1, PSB29, and LHCA6 were extremely high in the leaf area next to the main vein (Area 1), and decreased gradually from the middle to the edge of the leaf, indicating that the area next to the main vein might have a higher photosynthesis capacity compared with leaf edge. In addition, the significance of this spatial expression pattern and the precise spatial regulatory mechanism need to be 
371 further investigated. Meanwhile, we reconstructed those gene expression gradients to 372 show for the first time the developmental trajectories of vascular cells and guard cells 373 according to their spatial distribution. The tracking of spatial location of cells at various 374 developmental stages is meaningful. For example, spatial information combined cell type375 specific trajectory analysis could provide a reference for assessing the reliability of 376 development trajectory analysis results, and could facilitate researchers to sample cells at 377 specific developmental stages.

The scStereo-seq technology enabled us to reveal the complex cell type-specific and spatial-temporal gene expression features of Arabidopsis leaves. However, it is still in its infancy. First, the 871 single cells were manually extracted one by one using lasso tool in

382 this study, which was time- and labor-consuming. Therefore, we are also developing cell 383 boundary algorithms for automatic cell capture to meet the needs of high-throughput cell extraction for large tissues in future studies. Second, improvements in sectioning are required to obtain high-quality cell wall staining images. Because of the high water content in plants, cryosectioning is challenging for many plants or tissues. A high-quality slide is critical to identify all cell types in tissues. In this study, if high-quality slides were available, we were able to identify and extract multiple cell types of veins, which consist at least seven different cell types(Kim et al., 2021). Third, further improvement in the resolution of

390 the stereo-seq chips could facilitate identify tiny cells in certain tissues, such as plant shoot 391 apex.

393 Taking advantage of the bona fide single-cell spatial transcriptome, scStereo-seq has 394 great potential for future studies. For example, based on the high-resolution of scStereo395 seq, we would be able to analyze transcriptomes at subcellular level. Due to the simplicity 396 of scStereo-seq compared with MERFISH(Xia et al., 2019) and FISSEQ (fluorescent in 397 situ RNA sequencing)(Lee et al., 2014), it could be applied to broad plant research areas. 
We can also use scStereo-seq to analyze how different cell types respond to pathogen infection in plant leaf and root, and how response signals are transmitted to distant cells

400 and tissues. Moreover, scStereo-seq can be used to analyze the leaf differences between

401 C3 and C4 plants. By comparing transcriptome differences among bundle sheath cells, 402 other vascular cells and the surrounding mesophyll cells in C3 and C4 plants and 403 constructing developmental trajectories of bundle sheath cells, we could provide insights 404 for transforming C3 plants into C4 plants. In addition, scStereo-seq is also an ideal 405 technology to facilitate building Plant Cell Atlas(Rhee et al., 2019). The scStereo-seq 406 technology developed in this study offers a powerful single cell spatially resolved transcriptomic strategy for systematic studies of plant biology, and it will enable us to understand plants at unprecedented resolution.

\section{METHODS}

\section{Plant growth condition and tissue collection}

413 Arabidopsis (Col-0) seeds were sown and incubated at $25^{\circ} \mathrm{C}$ in the photoperiod of 16 414 hours light / 8 hours dark after surface sterilization with $8 \%$ sodium hypochlorite in $0.1 \%$ 415 triton X-100 solution. Fresh leaves were collected from five-week-old seedlings and 416 immediately fixed in Carnoy's fluid (3 ethanol: 1 acetic acid) for 20 minutes. After 10\% 417 glycerin treatment, tissues were embedded in cold OCT (Sakura) and stored at $-80{ }^{\circ} \mathrm{C}$ 418 until processed.

\section{Stereo-seq chip structure}

421 The Stereo-seq capture chips were used in this study(Chen A. et al., 2021). To generate 422 the DNA nanoball (DNB) array for in situ RNA capture, we first synthesized random 25-nt 423 CID (coordinate identity) -containing oligos, circularized with T4 ligase and splint oligos. 424 DNBs were then generated by rolling circle amplification and were loaded onto the 
425 patterned chips $(65 \mathrm{~mm} \times 65 \mathrm{~mm})$. The chip surface consists of DNA nanoball (DNB)

426 containing random barcoded sequences, the coordinate identity (CID), molecular

427 identifiers (MID) and polyT sequence-containing oligonucleotides. The DNBs are docked

428 in a grid-patterned array of spots, each spot being approximately $220 \mathrm{~nm}$ in diameter and

429 with a center-to-center distance of $715 \mathrm{~nm}$. Next, to determine the distinct DNB-CID

430 sequences at each spatial location, single-end sequencing was performed using

431 sequencing primers in a MGI DNBSEQ-Tx sequencer with sequencing strategy SE25.

432 Finally, MID and polyT-containing oligos were hybridized and ligated to the DNB on the

433 chip. This procedure produces capture probes containing a 25 bp CID barcode, a $10 \mathrm{bp}$

434 MID and a 22 bp polyT ready for in situ capture.

CID sequences together with their corresponding coordinates for each DNB were determined using a base calling method according to manufacturer's instruction of DNBSEQ ${ }^{\mathrm{TM}}$ sequencer. After sequencing, the capture chip was split into smaller size chips $(10 \mathrm{~mm} \times 10 \mathrm{~mm})$. At this stage, all duplicated CID that corresponded to non-adjacent 440 spots were filtered out.

\section{Cryosectioning, fixation, staining and imaging}

443 The pre-frozen leaf tissues in OCT were transversely sectioned at $10 \mu \mathrm{m}$ thickness using 444 a Leika CM1950 cryostat. Tissue sections were adhered to the Stereo-seq chip surface 445 and incubated at $37^{\circ} \mathrm{C}$ for 3 minutes. Then, tissues were fixed in methanol and incubated 446 at $-20{ }^{\circ} \mathrm{C}$ for 30 minutes. The same tissue sections were stained with toluidine blue for 447 stereo-seq, while tissue sections adjacent to those were adhered to glass slides for 448 histological examination using the same staining method. Imaging for both procedures was performed with a Ti-7 Nikon Eclipse microscope. 
preparation and sequencing

453 These processes were performed according to the previously reported Stereo-seq 454 method(Chen A. et al., 2021). Tissue patches on the chip were permeabilized using $0.1 \%$ 455 pepsin (Sigma, P7000) in $0.01 \mathrm{M} \mathrm{HCl}$ buffer $(\mathrm{pH}=2)$, incubated at $37^{\circ} \mathrm{C}$ for 10 minutes 456 and then washed with 0.1x SSC buffer (Thermo, AM9770) supplemented with $0.05 \mathrm{U} / \mu \mathrm{L}$ 457 RNase inhibitor (NEB, M0314L) after toluidine blue in tissues was rinsed off with $80 \%$ 458 ethanol. Released RNA from permeabilized tissues was captured by the DNB and reverse 459 transcribed overnight at $42{ }^{\circ} \mathrm{C}$ using SuperScript II (Invitrogen, 18064-014, $10 \mathrm{U} / \mu \mathrm{L}$ reverse transcriptase, $1 \mathrm{mM}$ dNTPs, $1 \mathrm{M}$ betaine solution PCR reagent, $7.5 \mathrm{mM} \mathrm{MgCl}_{2}, 5$

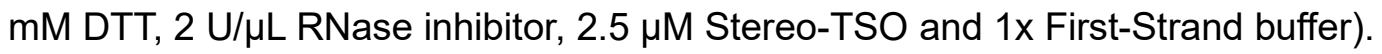

After in situ reverse transcription, tissue patches were washed twice with $0.1 \times$ SSC buffer and digested with Tissue Removal buffer (10 mM Tris- $\mathrm{HCl}, 25$ mM EDTA, $100 \mathrm{mM} \mathrm{NaCl}$, $0.5 \% \mathrm{SDS}$ ) at $37{ }^{\circ} \mathrm{C}$ for 30 minutes. cDNA-containing chips were then subjected to

466 Exonuclease I (NEB, M0293L) treatment for 3 hours at $37^{\circ} \mathrm{C}$ and were finally washed once with $0.1 \times$ SSC buffer. The resulting cDNAs released from chips were amplified with KAPA HiFi Hotstart Ready Mix (Roche, KK2602) with $0.8 \mu \mathrm{M}$ cDNA-PCR primer. PCR reactions were conducted as: first incubation at $95{ }^{\circ} \mathrm{C}$ for 5 minutes, 15 cycles at $98{ }^{\circ} \mathrm{C}$

470 for 20 seconds, $58{ }^{\circ} \mathrm{C}$ for 20 seconds, $72{ }^{\circ} \mathrm{C}$ for 3 minutes and a final incubation at $72{ }^{\circ} \mathrm{C}$ 471 for 5 minutes.

473 The concentrations of the resulting PCR products were quantified by Qubit ${ }^{\mathrm{TM}}$ dsDNA 474 Assay Kit (Thermo, Q32854). A total of 20 ng of DNA were then fragmented with in-house $475 \operatorname{Tn} 5$ transposase at $55^{\circ} \mathrm{C}$ for 10 minutes. Then, the reactions were stopped by the addition 476 of $0.02 \%$ SDS buffer and gently mixing at $37^{\circ} \mathrm{C}$ for 5 minutes. Fragmentation products 477 were amplified as described below: $25 \mu \mathrm{L}$ of fragmentation product, 1x KAPA HiFi Hotstart 478 Ready Mix and 0.3 $\mu \mathrm{M}$ Stereo-Library-F primer, $0.3 \mu \mathrm{M}$ Stereo-Library-R primer in a total 
479 volume of $100 \mu \mathrm{l}$ with the addition of nuclease-free $\mathrm{H}_{2} \mathrm{O}$. The reaction was then run as: 1

480 cycle of $95^{\circ} \mathrm{C}$ for 5 minutes, 13 cycle of $\left(98^{\circ} \mathrm{C} 20\right.$ seconds, $58^{\circ} \mathrm{C} 20$ seconds and $72^{\circ} \mathrm{C}$

48130 seconds) and 1 cycle $72{ }^{\circ} \mathrm{C}$ for 5 minutes. PCR products were purified using the 482 Ampure XP Beads (Vazyme, N411-03) (0.6x and 0.2x) for DNB generation and finally 483 sequenced (paired-end $50 \mathrm{bp}$ ) on a MGISEQ-2000RS sequencer.

\section{Raw Stereo-seq data processing}

486 Fastq files were generated using a MGISEQ-2000RS sequencer, and the raw data was 487 processed according to the Stereo-seq method(Chen A. et al., 2021). CID and MID are 488 contained in the forward reads (CID: 1-25 bp, MID: 26-35 bp) while the reverse reads 489 consist of the cDNA sequences. CID sequences on the forward reads were first mapped 490 to the designed coordinates of the in situ captured chip, allowing 1 base mismatch to 491 correct for sequencing and PCR errors. Reads with MID containing either $\mathrm{N}$ bases or more 492 than 2 bases with quality scores lower than 10 were filtered out. CID and MID associated 493 with each read were appended to each read header. Retained reads were then aligned to 494 Arabidopsis genome (TAIR10) using STAR(Dobin et al., 2013) and mapped reads with 495 MAPQ 10 were counted and annotated to their corresponding genes using an in-house 496 script (available at https://github.com/BGIResearch/handleBam). MID with the same CID 497 and the same gene locus were collapsed, allowing 1 mismatch to correct for sequencing 498 and PCR errors. Finally, this information was used to generate a CID-containing 499 expression profile matrix.

\section{Single cell data acquisition}

502 To obtain single-cell level transcriptome data, we used the lasso tool provided in the 503 visualization system of Stereomics to directly extract single-cell coordinate information and 504 MID count matrix according to the bright field below MID signals. With every cell extracted, 505 an information file was generated with bin1 size. We summed up the MID counts of the 
506

507

\section{Trajectory analysis}

532 Provided with spatial information of each single cell, the development direction of different 
533 cell types is certain, which is overall from Area 0 to 3 . We used the $\mathrm{R}$ package Monocle

534 (2.18.0)(Qiu et al., 2017) to carry out pseudotime analyses for vascular cell development

535 and epidermal cell differentiation. For the exploration of all vasculature-related cells in our

536 data, we selected DEGs between Area 0 and 3 cells that were detected in a minimum

537 fraction of 0.14 and p-value $<0.05$. The DEGs of epidermal cells and guard cells that were

538 detected in a minimum fraction of 0.08 and $p$-value $<0.05$ were chosen for the other

539 analysis. Then, we reduced our data to two components using the method "DDRTree".

540 Cells were ordered along the developmental paths and visualized in two-dimensional

541 space. Heatmaps were used to demonstrate the gene expression that differs in cells. The

542 data used in plotting the heat maps was subsequently used for GO biological process

543 analysis.

GO enrichment analysis

546 GO enrichment analysis was performed using R package clusterProfiler(Yu et al., 2012)

547 with TAIR10 annotation as the background. The smaller the $P$-value is, the more the GO 548 term is significantly enriched.

\section{ACKNOWLEDGMENTS}

551 This research was supported by Guangdong Provincial Key Laboratory of Genome Read 552 and Write (No. 2017B030301011), and Shenzhen Key Laboratory of Single-Cell Omics 553 (No. ZDSYS20190902093613831). We also sincerely thank the support provided by 554 China National GeneBank (CNGB).

\section{AUTHOR CONTRIBUTIONS}

557 X.X., Y.G., J.W., K.X., and H.-X.S. designed and supervised the study. K.X., H.-X.S., and 558 Y.Z. designed the experiment. Y.Z., G.L., Z.C., and R.Y. performed the library preparation 559 and sequencing. J.L., R.C., S.H. and H.-X.S. performed bioinformatics analysis. J.X. 
560 provided technical support. J.W., Q.X., Y.L., A.C., L.L., Y.Y., and H.Y. gave the relevant 561 advice. K.X., H.-X.S., J.-M.L. J.L., G.L., Z.C. and R.C. wrote the manuscript. K.X., H.-X.S., 562 X. X. and Y. G. participated in the manuscript editing and discussion. All authors edited 563 and approved the manuscript.

565 DECLARATION OF INTERESTS

566 The authors declare the following competing interests: The chip, procedure and 567 applications of Stereo-seq are covered in pending patents. Employees of BGI have stock 568 holdings in BGI.

\section{DATA AVAILABILITY}

571 The high-throughput sequencing data that support the findings of this study have been 572 deposited into CNGB Sequence Archive(Guo et al., 2020) of CNGBdb(Chen et al., 2020) 573 with accession number CNP0001923. 
578 Figure 1. Single-cell level of spatially resolved transcriptome in Arabidopsis 579 thaliana cauline leaves

580 (A) Schematic representation of the single-cell Stereo-seq procedure. A. thaliana cauline 581 leaves are cryosectioned and positioned on top of the chip surface with DNA nanoball 582 (DNB) docked in a grid-patterned array of spots, and capture probes contain CID 583 (coordinate identity) barcode, MID (molecular identifiers) and PloyT oligos to enable the 584 recordation of the spatial coordinates, the identification of unique transcripts per gene, and 585 the capture of mRNAs. After cell wall staining and imaging, the same section is sequenced with Stereo-seq. Through the combined high-resolution image and molecular identifiers 587 (MID), single-cell level of MID distribution is achieved. The lasso tool is used to manually extract single cells, and major cell types of cauline leaves are identified and mapped back to the bright field image. Using the spatial single-cell data, several cell sub-types are distinguished (i). And next, the leaf is divided into four distinct parts and spatial gene expression pattern (ii) and spatial developmental trajectory (iii) are determined. distribution in the leaf. The color bar represents the number of MIDs. The scale bar is 500 $594 \mu \mathrm{m}$.

(C-D) Violin plots represent the number of genes and MIDs per cell/bin, and the percentage of mitochondria and chloroplast genes per cell/bin of sequenced cells/bins in all cauline leaves (C) and in different cauline leaves (D). The black diamond indicates the median number of genes/MIDs or percentage of mitochondria genes/chloroplast genes per cell/bin. Asterisks indicate statistically significant differences: $\left({ }^{* * *}\right) P<0.001$. 
603 (A) Distribution of different cell types in the bright field picture. The scale bar is $500 \mu \mathrm{m}$.

604 (B) Violin plots show the gene number, the MID number, the percentage of mitochondria 605 genes, and the percentage of chloroplast genes per cell in different cell types. The black 606 diamond indicates the median number of genes/MIDs or percentage of mitochondria 607 genes/chloroplast genes per cell.

608 (C) Dot plots showing the top five up-regulated marker genes of each cell type. The circle 609 size indicates the percentage of cells expressing the marker genes, while color represents 610 expression value.

611 (D) Percentage of cells/bins expressing cell-type markers.

612 (E) PCA plot of vascular cells and mesophyll cells in different leaves (left) and heatmap 613 showing DEGs between vascular cells and mesophyll cells (right). Blue and red represent $614 \log _{2}$-transformed fold change $<0$ and $>0$, respectively. Asterisks indicate statistically 615 significant differences: $\left.{ }^{* * *}\right) \mathrm{P}<0.001$.

Figure 3. Validation and investigation of transcriptome characteristics of cell types and cell sub-types

620 Representative images showing expression levels of marker genes in the corresponding 621 cells in the bright field (lower panel).

622 (B) Enriched GO terms for four different cell types.

623 (C) scStereo-seq allows separation between upper and lower epidermal cells. Distribution 624 of upper and lower epidermal cells: i) without spatial information (using variable genes of 625 all epidermal cells); and ii) with spatial information (using DEGs between upper and lower 626 epidermal cells). Blue dots represent upper epidermal cells and orange dots represent 627 lower epidermal cells.

628 (D) DEGs between upper and lower epidermal cells. Blue and red represent $\log _{2^{-}}$ 629 transformed fold change $<0$ and $>0$, respectively. 
630 (E) GO enrichment analysis of DEGs between upper and lower epidermal cells.

632 Figure 4. Spatial distribution of photosynthesis-related genes in Arabidopsis leaves

633 (A) Four unique areas (Area 0, 1, 2, 3) from main vein to leaf edge in a leaf.

634 (B) Gene expression patterns for all cells.

635 (C) GO enrichment analysis for genes present in each pattern.

636 (D) Dot plot showing expression levels of representative photosynthesis-related genes in 637 all cells.

638 (E) Gene expression patterns for mesophyll cells.

639 (F) GO enrichment analysis for genes present in each pattern.

640 (G) Dot plot showing expression levels of representative photosynthesis-related genes in 641 mesophyll cells. For dot plots the circle size indicates the percentage of cells expressing 642 the marker genes, while color represents expression value. For pattern analysis, only 643 genes with membership $>0.41$ are shown.

Figure 5. Spatially resolved developmental trajectories of vascular cells and guard cells

647 (A) Distribution of vascular cells on the pseudotime trajectory (top); distribution of vascular 648 cells from each area on the pseudotime trajectory (bottom).

649 (B) Clustering of differentially expressed genes along a pseudo-time progression of 650 vascular cells (left); top five enriched GO terms for each cluster (right).

651 (C) Gene expression kinetics along pseudotime progression for representative genes; 652 each dot represents one cell and dot color represents its location.

653 (D) Distribution of guard cells and epidermal cells on the pseudotime trajectory branches, 654 including pre-branch, cell fate 1 and cell fate 2.

655 (E) Proportion of guard cells in each branch.

656 (F) Clustering of differentially expressed genes along a pseudotime progression of 
657 epidermal cells and guard cells (left); representative enriched GO terms for each cluster 658 (right). N.S. represents not significant.

659

660

\section{1}

662

663

664

665

666

667

668

\section{Supplementary Information}

Figure S1. MID distribution in 11 Arabidopsis thaliana cauline leaves, Related to

\section{Figure 1}

(A and B) Bright field image and MID distribution in Arabidopsis cauline leaves from Section 1 (S1, A) and Section 2 (S2, B). The scale bar is $500 \mu \mathrm{m}$. The color bar represents the number of MIDs.

(C) Scatterplot showing gene number and MID number per cell in 11 leaves. All points are summarized and shown as density plots along each axis.

\section{Figure S2. Comparison of transcriptome profiles between bins and single cells and} technical reproducibility of cell types from different cauline leaves, Related to Figure 2

(A-D) Percentage of cells/bins expressing markers of epidermal cells (A), mesophyll cells (B), vascular cells (C) and guard cells (D).

(E-I) PCA plot of vascular cells and epidermal cells (E), vascular cells and guard cells (F), epidermal cells and mesophyll cells $(G)$, mesophyll cells and guard cells $(H)$, and epidermal cells and guard cells (I) in different leaves (left). The heatmaps showing DEGs between the above cell types are shown on the right. Asterisks indicate statistically significant differences: $\left({ }^{* *}\right) P<0.001$. 
684 (A) Validation of cell types using cell-type specific marker genes (top panel).

685 Representative images showing expression levels of marker genes in the corresponding 686 cells in the bright field (lower panel).

687 (B) Spatial information allows separation between palisade and spongy mesophyll cells.

688 Distribution of palisade and spongy mesophyll cells: i) without spatial information (using 689 variable genes of all mesophyll cells); and ii) with spatial information (using DEGs between 690 palisade and spongy mesophyll cells). Green dots represent palisade mesophyll cell and 691 yellow dots represent spongy mesophyll cell.

692 (C) DEGs between palisade mesophyll cell and spongy mesophyll cell. Blue and red 693 represent $\log _{2}$-transformed fold change $<0$ and $>0$, respectively.

694 (D) GO enrichment analysis of DEGs between palisade mesophyll cell and spongy 695 mesophyll cell.

696 (E) UMAP projection plot of bins from epidermal cells using: i) variable genes of bins from 697 epidermal cells; and ii) differentially expressed genes (DEGs) between bins from upper 698 and lower epidermal cells.

699 (F) Only 21 DEGs were overlapped between upper and lower epidermal cells using single700 cell method and Bin20 method.

701

Figure S4. Spatially resolved expression patterns of vascular cells and spatial distribution of guard cells and epidermal cells, Related to Figure 5

(A) Gene expression kinetics along pseudotime progression for representative genes from vascular cells.

707 trajectory.

708 (C) Statistics of spatial distribution of guard cells and epidermal cells in each area from 709 each branch. 
bioRxiv preprint doi: https://doi.org/10.1101/2021.10.20.465066; this version posted October 21, 2021. The copyright holder for this preprint (which was not certified by peer review) is the author/funder. All rights reserved. No reuse allowed without permission.

711 Table S1 Statistics of single cells detected by scStereo-seq from 11 cauline leaves,

712 Related to Figure 1

713

714 
REFERENCES

716

717

718

719

720

721

722

723

724

725

726

727

728

729

730

731

732

733

734

735

736

737

738

739

740

741

742

743

744

745

746

747

748

749

750

751

752

753

754

Bezrutczyk, M., Zollner, N.R., Kruse, C.P.S., Hartwig, T., Lautwein, T., Kohrer, K., Frommer, W.B., and Kim, J.Y. (2021). Evidence for phloem loading via the abaxial bundle sheath cells in maize leaves. Plant Cell 33, 531547.

Brown, N.J., Palmer, B.G., Stanley, S., Hajaji, H., Janacek, S.H., Astley, H.M., Parsley, K., Kajala, K., Quick, W.P., Trenkamp, S., et al. (2010). C acid decarboxylases required for C photosynthesis are active in the mid-vein of the $\mathrm{C}$ species Arabidopsis thaliana, and are important in sugar and amino acid metabolism. Plant J 61, 122-133.

Chen A., Chen A., Chen A., Chen A., Chen A., Chen A., and Chen A. (2021). Large field of view-spatially resolved transcriptomics at nanoscale resolution. bioRxiv.

Chen, F.Z., You, L.J., Yang, F., Wang, L.N., Guo, X.Q., Gao, F., Hua, C., Tan, C., Fang, L., Shan, R.Q., et al. (2020). CNGBdb: China National GeneBank DataBase. Yi Chuan 42, 799-809.

Chen, K.H., Boettiger, A.N., Moffitt, J.R., Wang, S., and Zhuang, X. (2015). RNA imaging. Spatially resolved, highly multiplexed RNA profiling in single cells. Science 348 , aaa6090.

Cho, C.S., Xi, J., Si, Y., Park, S.R., Hsu, J.E., Kim, M., Jun, G., Kang, H.M., and Lee, J.H. (2021). Microscopic examination of spatial transcriptome using Seq-Scope. Cell 184, 3559-3572 e3522.

Dobin, A., Davis, C.A., Schlesinger, F., Drenkow, J., Zaleski, C., Jha, S., Batut, P., Chaisson, M., and Gingeras, T.R. (2013). STAR: ultrafast universal RNA-seq aligner. Bioinformatics (Oxford, England) 29, 15-21.

Eng, C.L., Lawson, M., Zhu, Q., Dries, R., Koulena, N., Takei, Y., Yun, J., Cronin, C., Karp, C., Yuan, G.C., et al. (2019). Transcriptome-scale super-resolved imaging in tissues by RNA seqFISH. Nature 568, 235-239.

Frenette Charron, J.B., Breton, G., Badawi, M., and Sarhan, F. (2002). Molecular and structural analyses of a novel temperature stress-induced lipocalin from wheat and Arabidopsis. FEBS Lett 517, 129-132.

Gao, Z., Shen, W., and Chen, G. (2018). Uncovering C4-like photosynthesis in C3 vascular cells. J Exp Bot 69, 3531-3540.

Geng, P., Zhang, S., Liu, J., Zhao, C., and Zhao, Q. (2019). MYB20, MYB42, MYB43 and MYB85 Regulate Phenylalanine and Lignin Biosynthesis during Secondary Cell Wall Formation. Plant Physiology 182, pp.01070.02019.

Giacomello, S., Salmen, F., Terebieniec, B.K., Vickovic, S., Navarro, J.F., Alexeyenko, A., Reimegard, J., McKee, L.S., Mannapperuma, C., Bulone, V., et al. (2017). Spatially resolved transcriptome profiling in model plant species. Nat Plants 3, 17061.

Glover, B.J., Airoldi, C.A., and Moyroud, E. (2016). Epidermis: Outer Cell Layer of the Plant. eLS.

Guo, X., Chen, F., Gao, F., Li, L., Liu, K., You, L., Hua, C., Yang, F., Liu, W., Peng, C., et al. (2020). CNSA: a data repository for archiving omics data. Database (Oxford) 2020.

Gurazada, S.G.R., Cox, K.L., Czymmek, K.J., and Meyers, B.C. (2021). Space: the final frontier - achieving single-cell, spatially resolved transcriptomics in plants. Emerg Top Life Sci.

Hibberd, J.M., and Quick, W.P. (2002). Characteristics of C4 photosynthesis in stems and petioles of C3 flowering plants. Nature 415, 451-454.

Ischebeck, T., Werner, S., Krishnamoorthy, P., Lerche, J., Meijon, M., Stenzel, I., Lofke, C., Wiessner, T., Im, Y.J., Perera, I.Y., et al. (2013). Phosphatidylinositol 4,5-bisphosphate influences PIN polarization by 
controlling clathrin-mediated membrane trafficking in Arabidopsis. The Plant cell 25, 4894-4911.

Ishihara, S., Takabayashi, A., Ido, K., Endo, T., Ifuku, K., and Sato, F. (2007). Distinct functions for the two PsbP-like proteins PPL1 and PPL2 in the chloroplast thylakoid lumen of Arabidopsis. Plant Physiol 145, 668679.

Jiao, Y., Tausta, S.L., Gandotra, N., Sun, N., Liu, T., Clay, N.K., Ceserani, T., Chen, M., Ma, L., Holford, M., et al. (2009). A transcriptome atlas of rice cell types uncovers cellular, functional and developmental hierarchies. Nat Genet 41, 258-263.

Kawamura, Y., and Uemura, M. (2003). Mass spectrometric approach for identifying putative plasma membrane proteins of Arabidopsis leaves associated with cold acclimation. Plant J 36, 141-154.

Keren, N., Ohkawa, H., Welsh, E.A., Liberton, M., and Pakrasi, H.B. (2005). Psb29, a conserved 22-kD protein, functions in the biogenesis of Photosystem II complexes in Synechocystis and Arabidopsis. Plant Cell 17, 2768-2781.

Kim, J.-Y., Kim, J.-Y., Kim, J.-Y., Kim, J.-Y., Kim, J.-Y., Kim, J.-Y., Kim, J.-Y., and Kim, J.-Y. (2021). Distinct identities of leaf phloem cells revealed by single cell transcriptomics. Plant Cell.

Kimura, M., Yamamoto, Y.Y., Seki, M., Sakurai, T., Sato, M., Abe, T., Yoshida, S., Manabe, K., Shinozaki, K., and Matsui, M. (2003). Identification of Arabidopsis genes regulated by high light-stress using cDNA microarray. Photochem Photobiol 77, 226-233.

Kubo, M., Nishiyama, T., Tamada, Y., Sano, R., Ishikawa, M., Murata, T., Imai, A., Lang, D., Demura, T., Reski, R., et al. (2019). Single-cell transcriptome analysis of Physcomitrella leaf cells during reprogramming using microcapillary manipulation. Nucleic Acids Res 47, 4539-4553.

Lee, J.H., Daugharthy, E.R., Scheiman, J., Kalhor, R., Yang, J.L., Ferrante, T.C., Terry, R., Jeanty, S.S., Li, C., Amamoto, R., et al. (2014). Highly multiplexed subcellular RNA sequencing in situ. Science 343, 1360-1363. Liu, Y., Yang, M., Deng, Y., Su, G., Enninful, A., Guo, C.C., Tebaldi, T., Zhang, D., Kim, D., Bai, Z., et al. (2020a). High-Spatial-Resolution Multi-Omics Sequencing via Deterministic Barcoding in Tissue. Cell 183, 1665-1681 e1618.

Liu, Z., Zhou, Y., Guo, J., Li, J., Tian, Z., Zhu, Z., Wang, J., Wu, R., Zhang, B., Hu, Y., et al. (2020b). Global Dynamic Molecular Profiling of Stomatal Lineage Cell Development by Single-Cell RNA Sequencing. Mol Plant 13, 1178-1193.

Lopez-Anido, C.B., Vaten, A., Smoot, N.K., Sharma, N., Guo, V., Gong, Y., Anleu Gil, M.X., Weimer, A.K., and Bergmann, D.C. (2021). Single-cell resolution of lineage trajectories in the Arabidopsis stomatal lineage and developing leaf. Dev Cell 56, 1043-1055 e1044.

Martinez, C.C., Li, S., Woodhouse, M.R., Sugimoto, K., and Sinha, N.R. (2021). Spatial transcriptional signatures define margin morphogenesis along the proximal-distal and medio-lateral axes in tomato (Solanum lycopersicum) leaves. Plant Cell 33, 44-65.

Maugarny-Cales, A., and Laufs, P. (2018). Getting leaves into shape: a molecular, cellular, environmental and evolutionary view. Development 145.

Miki, Y., Takahashi, D., Kawamura, Y., and Uemura, M. (2019). Temporal proteomics of Arabidopsis plasma membrane during cold- and de-acclimation. J Proteomics 197, 71-81.

Morales, R., Charon, M.H., Kachalova, G., Serre, L., Medina, M., Gomez-Moreno, C., and Frey, M. (2000). A redox-dependent interaction between two electron-transfer partners involved in photosynthesis. EMBO Rep 1, 271-276. 
Nichterwitz, S., Chen, G., Aguila Benitez, J., Yilmaz, M., Storvall, H., Cao, M., Sandberg, R., Deng, Q., and Hedlund, E. (2016). Laser capture microscopy coupled with Smart-seq2 for precise spatial transcriptomic profiling. Nat Commun 7, 12139.

Peng, L., Fukao, Y., Fujiwara, M., Takami, T., and Shikanai, T. (2009). Efficient operation of NAD(P)H dehydrogenase requires supercomplex formation with photosystem I via minor $\mathrm{LHCl}$ in Arabidopsis. Plant Cell 21, 3623-3640.

Qiu, X., Mao, Q., Tang, Y., Wang, L., Chawla, R., Pliner, H.A., and Trapnell, C. (2017). Reversed graph embedding resolves complex single-cell trajectories. Nat Methods 14, 979-982.

Rhee, S.Y., Birnbaum, K.D., and Ehrhardt, D.W. (2019). Towards Building a Plant Cell Atlas. Trends Plant Sci 24, 303-310.

Rich-Griffin, C., Stechemesser, A., Finch, J., Lucas, E., Ott, S., and Schafer, P. (2020). Single-Cell Transcriptomics: A High-Resolution Avenue for Plant Functional Genomics. Trends Plant Sci 25, 186-197.

Rodriques, S.G., Stickels, R.R., Goeva, A., Martin, C.A., Murray, E., Vanderburg, C.R., Welch, J., Chen, L.M., Chen, F., and Macosko, E.Z. (2019). Slide-seq: A scalable technology for measuring genome-wide expression at high spatial resolution. Science 363, 1463-1467.

Ruan, Y.L. (2014). Sucrose metabolism: gateway to diverse carbon use and sugar signaling. Annu Rev Plant Biol 65, 33-67.

Shaw, R., Tian, X., and Xu, J. (2021). Single-Cell Transcriptome Analysis in Plants: Advances and Challenges. Mol Plant 14, 115-126.

Srivatsan, S.R., Regier, M.C., Barkan, E., Franks, J.M., Packer, J.S., Grosjean, P., Duran, M., Saxton, S., Ladd, J.J., Spielmann, M., et al. (2021). Embryo-scale, single-cell spatial transcriptomics. Science 373, 111-117. Stahl, P.L., Salmen, F., Vickovic, S., Lundmark, A., Navarro, J.F., Magnusson, J., Giacomello, S., Asp, M., Westholm, J.O., Huss, M., et al. (2016). Visualization and analysis of gene expression in tissue sections by spatial transcriptomics. Science 353, 78-82.

Stickels, R.R., Murray, E., Kumar, P., Li, J., Marshall, J.L., Di Bella, D.J., Arlotta, P., Macosko, E.Z., and Chen, F. (2021). Highly sensitive spatial transcriptomics at near-cellular resolution with Slide-seqV2. Nat Biotechnol 39, 313-319.

Svozil, J., Gruissem, W., and Baerenfaller, K. (2015). Proteasome targeting of proteins in Arabidopsis leaf mesophyll, epidermal and vascular tissues. Front Plant Sci 6, 376.

Tian, C., Du, Q., Xu, M., Du, F., and Jiao, Y. (2020). Single-nucleus RNA-seq resolves spatiotemporal developmental trajectories in the tomato shoot apex. bioRxiv.

Tian, C., Wang, Y., Yu, H., He, J., Wang, J., Shi, B., Du, Q., Provart, N.J., Meyerowitz, E.M., and Jiao, Y. (2019). A gene expression map of shoot domains reveals regulatory mechanisms. Nat Commun 10, 141. Tsukaya, H. (2013). Leaf development. Arabidopsis Book 11, e0163.

Vickovic, S., Eraslan, G., Salmen, F., Klughammer, J., Stenbeck, L., Schapiro, D., Aijo, T., Bonneau, R., Bergenstrahle, L., Navarro, J.F., et al. (2019). High-definition spatial transcriptomics for in situ tissue profiling. Nat Methods 16, 987-990.

Wang, J., Sun, N., Zhang, F., Yu, R., Chen, H., Deng, X.W., and Wei, N. (2020). SAUR17 and SAUR50 Differentially Regulate PP2C-D1 during Apical Hook Development and Cotyledon Opening in Arabidopsis. Plant Cell 32, 3792-3811.

Wang, X., Allen, W.E., Wright, M.A., Sylwestrak, E.L., Samusik, N., Vesuna, S., Evans, K., Liu, C., Ramakrishnan, 
837 C., Liu, J., et al. (2018). Three-dimensional intact-tissue sequencing of single-cell transcriptional states.

838 Science 361.

839 Xia, C., Fan, J., Emanuel, G., Hao, J., and Zhuang, X. (2019). Spatial transcriptome profiling by MERFISH

840 reveals subcellular RNA compartmentalization and cell cycle-dependent gene expression. Proc Natl Acad Sci

841 U S A 116, 19490-19499.

842 Yabuta, S., Ifuku, K., Takabayashi, A., Ishihara, S., Ido, K., Ishikawa, N., Endo, T., and Sato, F. (2010). Three

843 PsbQ-like proteins are required for the function of the chloroplast NAD(P)H dehydrogenase complex in

844 Arabidopsis. Plant Cell Physiol 51, 866-876.

845 Yu, G., Wang, L.G., Han, Y., and He, Q.Y. (2012). clusterProfiler: an R package for comparing biological themes

846 among gene clusters. OMICS 16, 284-287.

847 Zhang, T.Q., Chen, Y., and Wang, J.W. (2021). A single-cell analysis of the Arabidopsis vegetative shoot apex.

848 Dev Cell 56, 1056-1074 e1058.

849 
Goppivpreprindoi: tps://doi.org/10.1101/2021.10.20.465066; this version posted October 21, 2021. The copyright holder for this preprint A

scStereo-seq
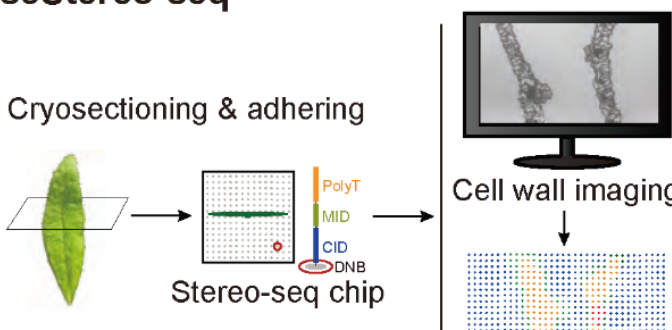

Cell wall imaging

$\downarrow$

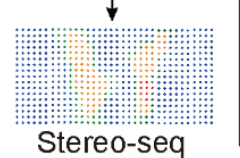

Single cell data

acquisition

Stereo-seq
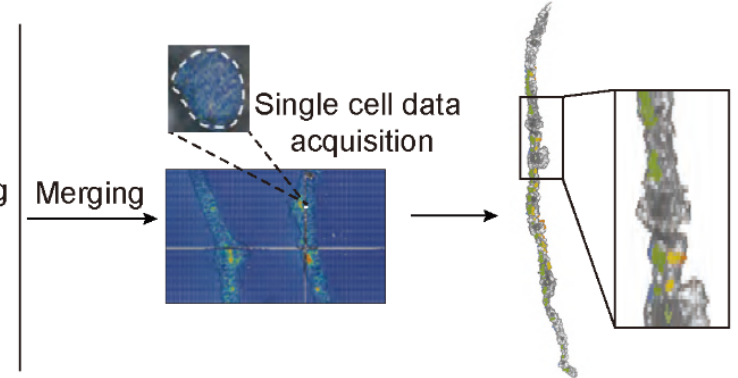

Epidermal cell:

Upper epidermal cell

Lower epidermal cell

Mesophyll cell:

Palisade mesophyll cell

Spongy mesophyll cell

- Guard cell

Spatial single-cell transcriptome analysis

(i) Cell sub-type identification

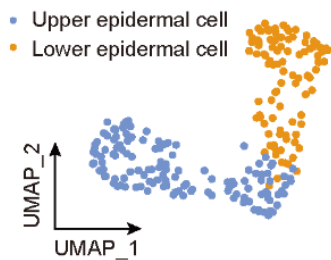

(ii) Spatial gene expression pattern

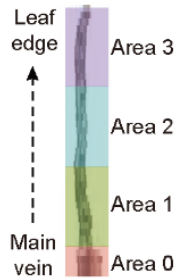

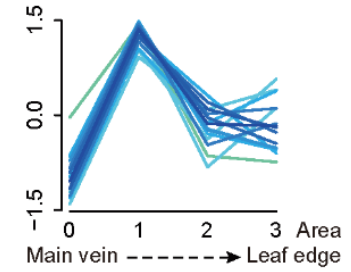

(iii) Spatial developmental trajectory

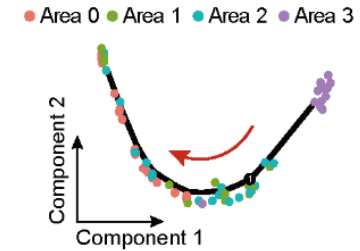

B

Bright field (S1-2)

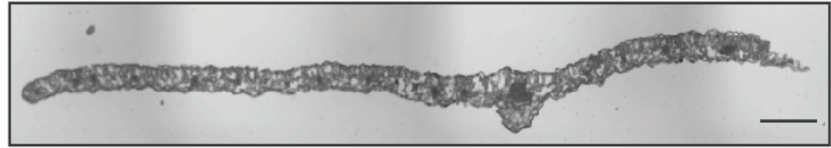

MID distribution (S1-2)

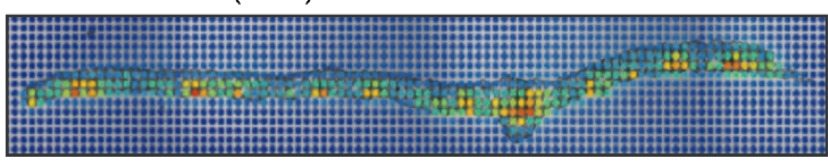

MID number

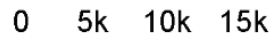

D

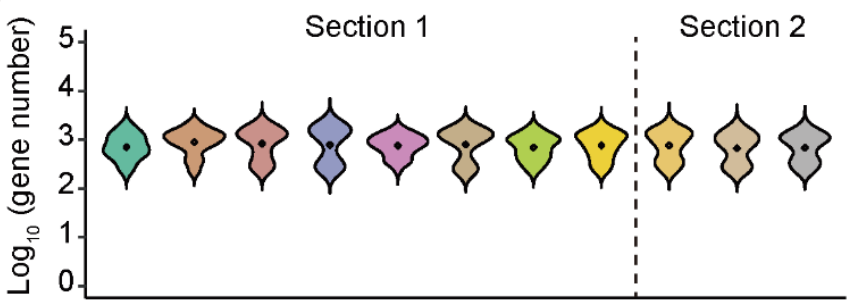

C

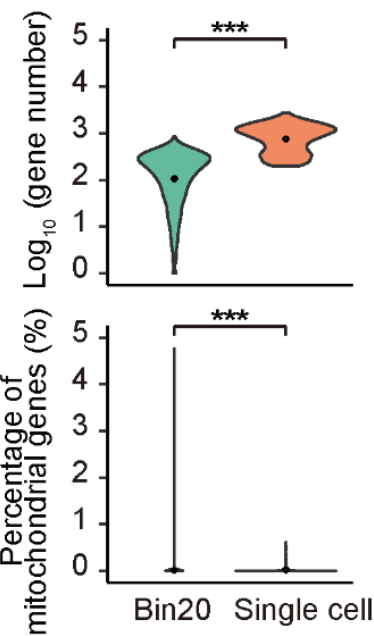

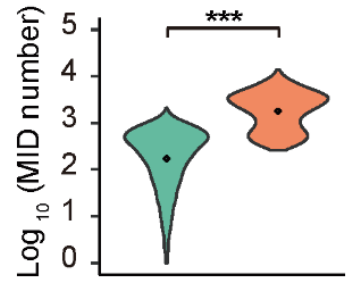

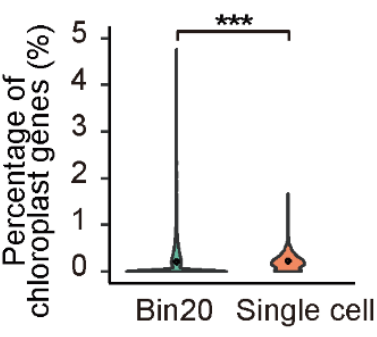

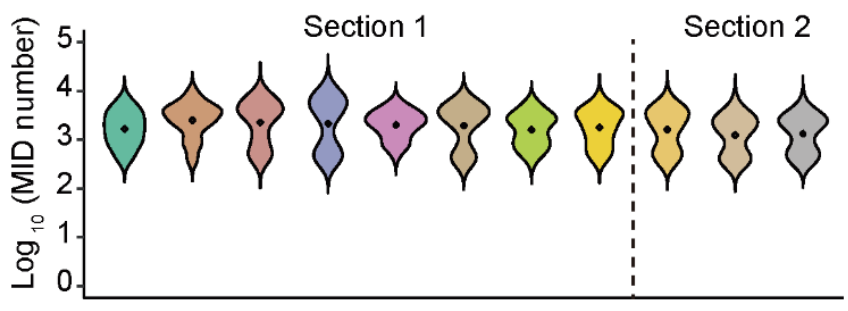

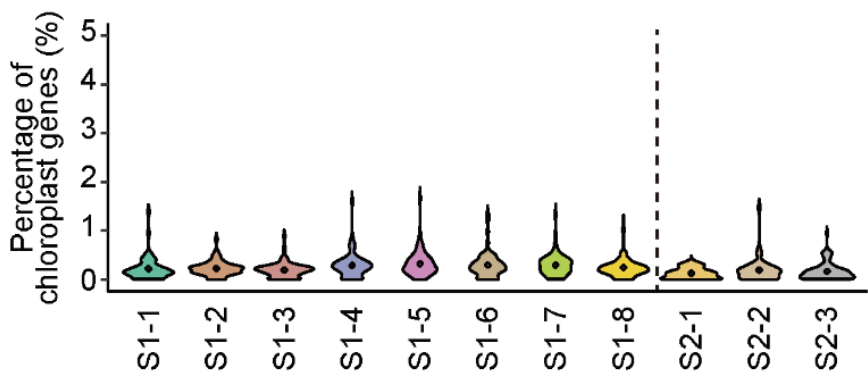


A

$\mathrm{S} 2-1$

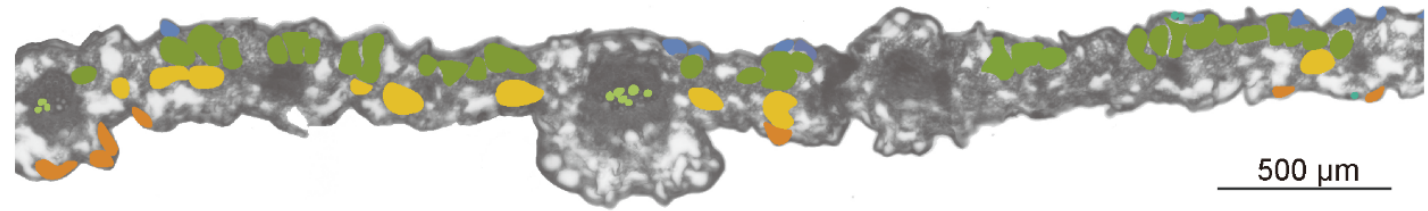

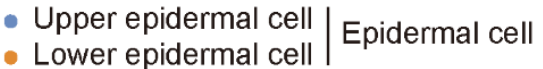

\begin{tabular}{l|l} 
- Palisade mesophyll cell & Mesophyll cell \\
- Spongy mesophyll cell &
\end{tabular}

- Guard cell

- Vascular cell

B
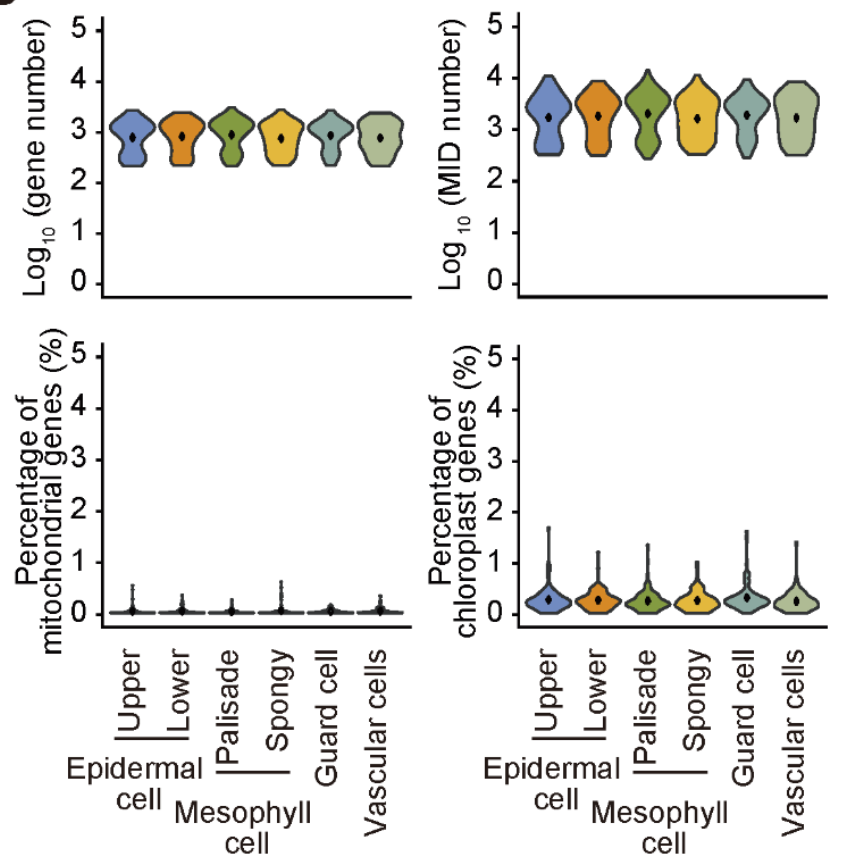

D

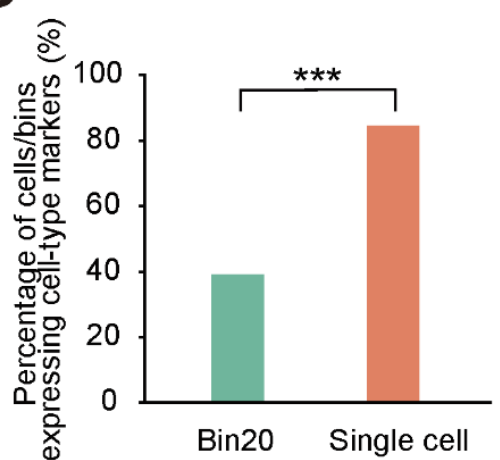

E
C

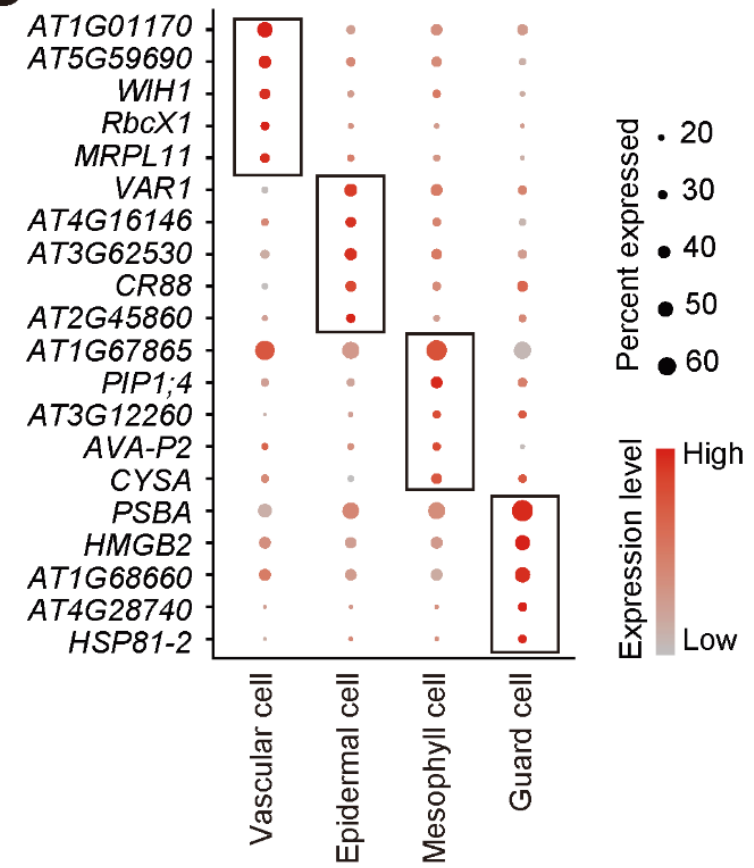

- Vascular cells - Mesophyll cells

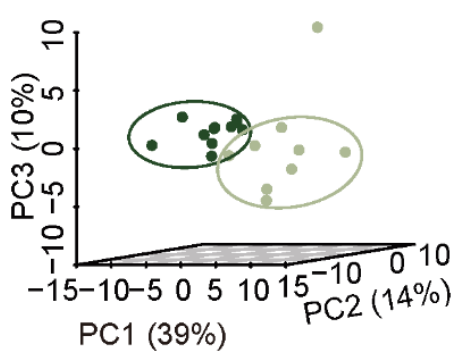

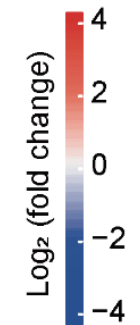




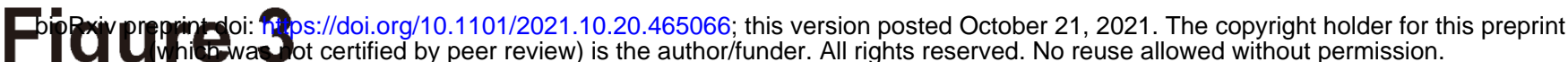
A
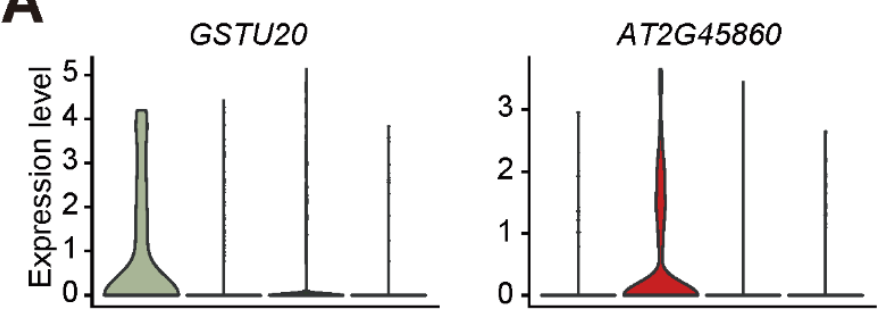

AT2G45860
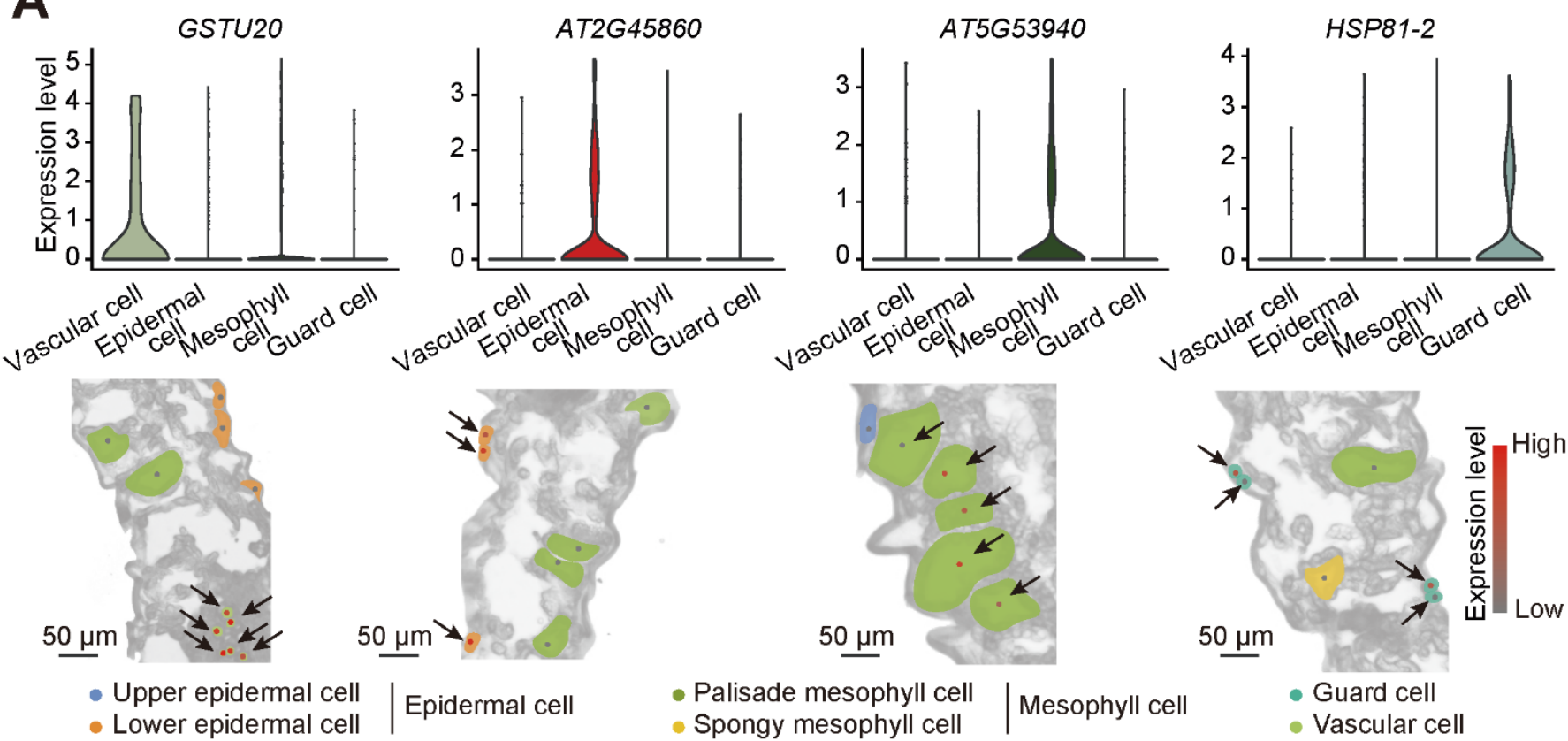

B

\begin{tabular}{l|l} 
- Upper epidermal cell & Epidermal cell
\end{tabular}

\begin{tabular}{l|l} 
- Palisade mesophyll cell & Mesophyll cell \\
- Spongy mesophyll cell
\end{tabular}

- Vascular cell

C

Photomorphogenesis Photoinhibition

Negative regulation of photosynthesis, light reaction Negative regulation of photosynthesis

Pathogenesis

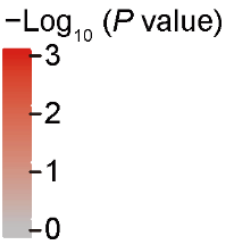

Circadian rhythm

Rhythmic process

Response to cadmium ion

Response to high light intensity

Regulation of starch metabolic process

Sucrose metabolic process

Photosystem

Photosynthesis, light harvesting in photosystem I

Photosynthesis, light harvesting

Positive regulation of ion transport

Regulation of microtubule polymerization or depolymerization

Regulation of metal ion transport

Iron ion transport

Response to starvation

Response to iron ion starvation

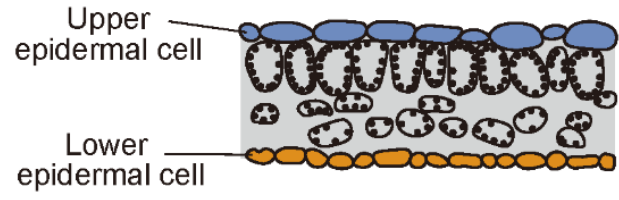

i) Single cell transcriptome alone

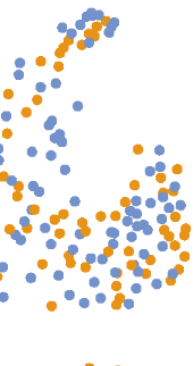

ii) Single cell transcriptome + spatial information
D

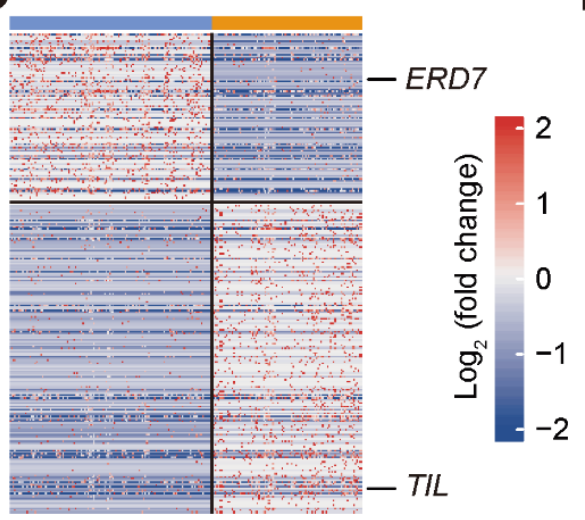

Drought recovery

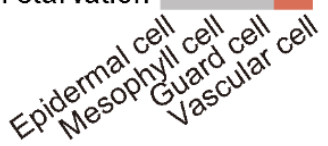

E

- Upper epidermal cell $\quad$ Lower epidermal cell

Response to high light intensity

Phenol-containing compound metabolic process

Secondary metabolic process

Response to insect

Wax biosynthetic process

Response to cold

Response to freezing

Membrane lipid metabolic process

Regulation of programmed cell death

Fatty acid metabolic process

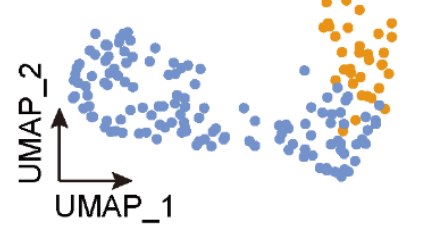




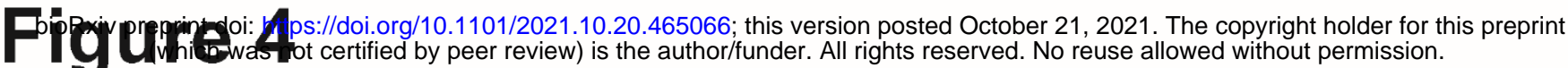

A

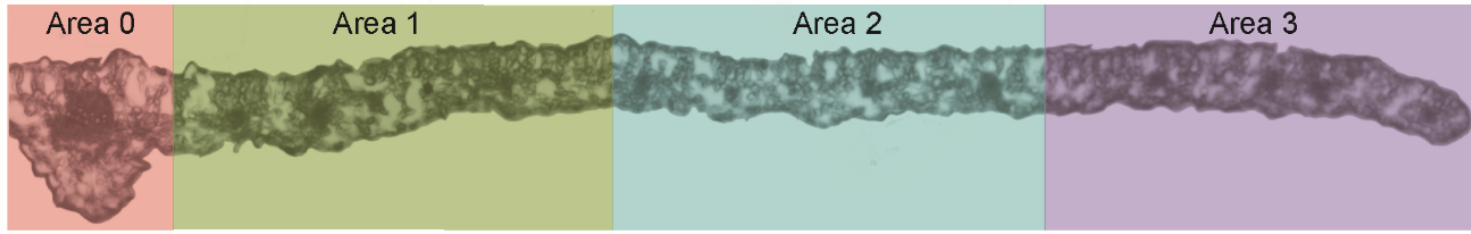

Main vein $------------------------\rightarrow$ Leaf edge

B

All cells

Pattern 1
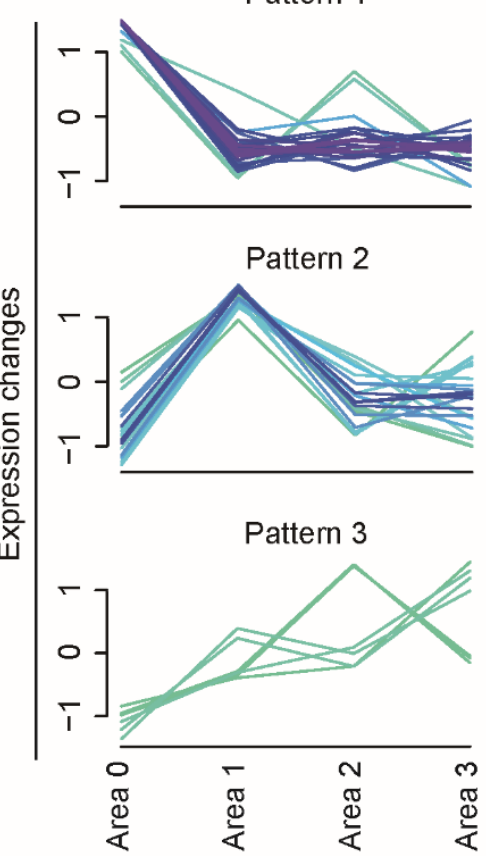

E

Mesophyll cells

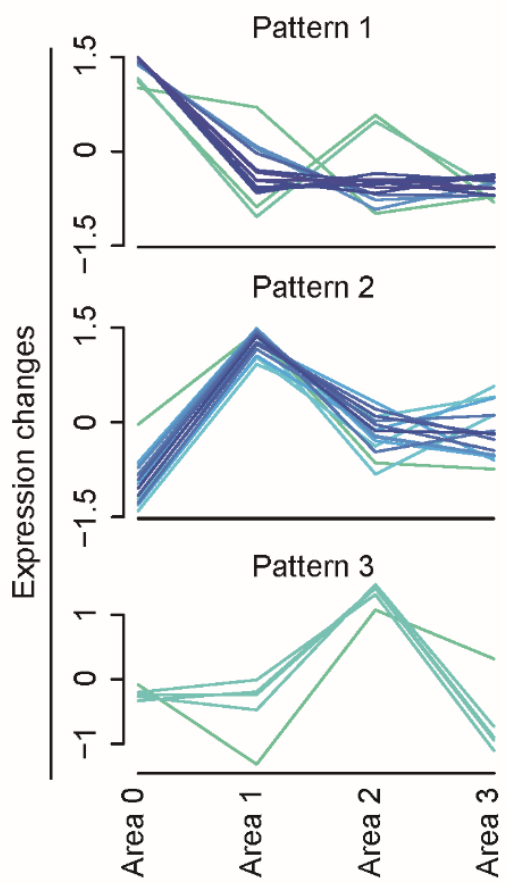

All cells

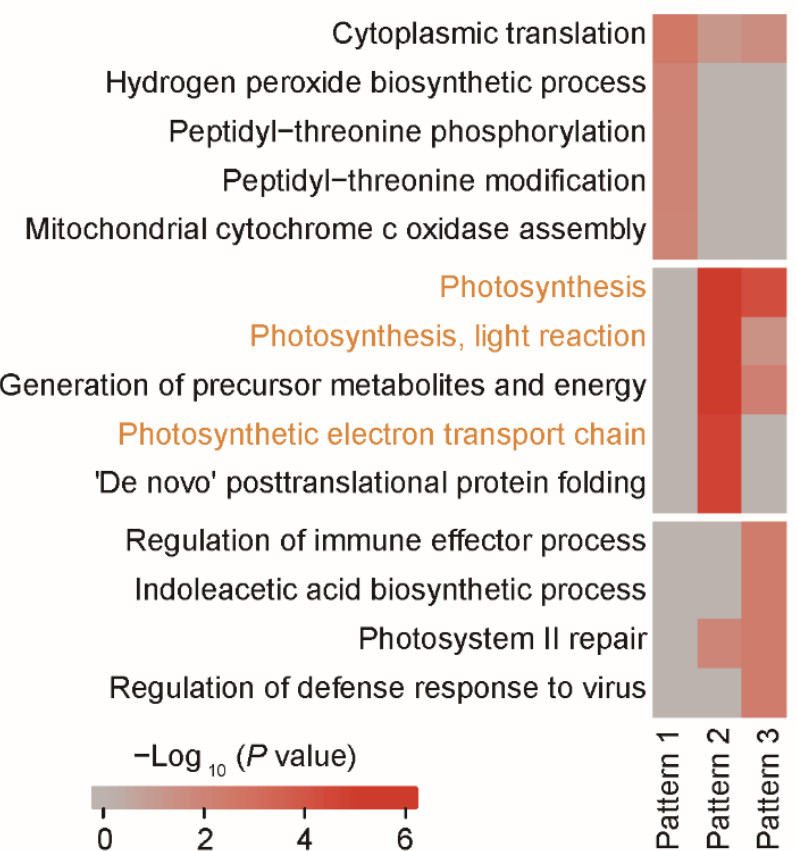

F

Mesophyll cells

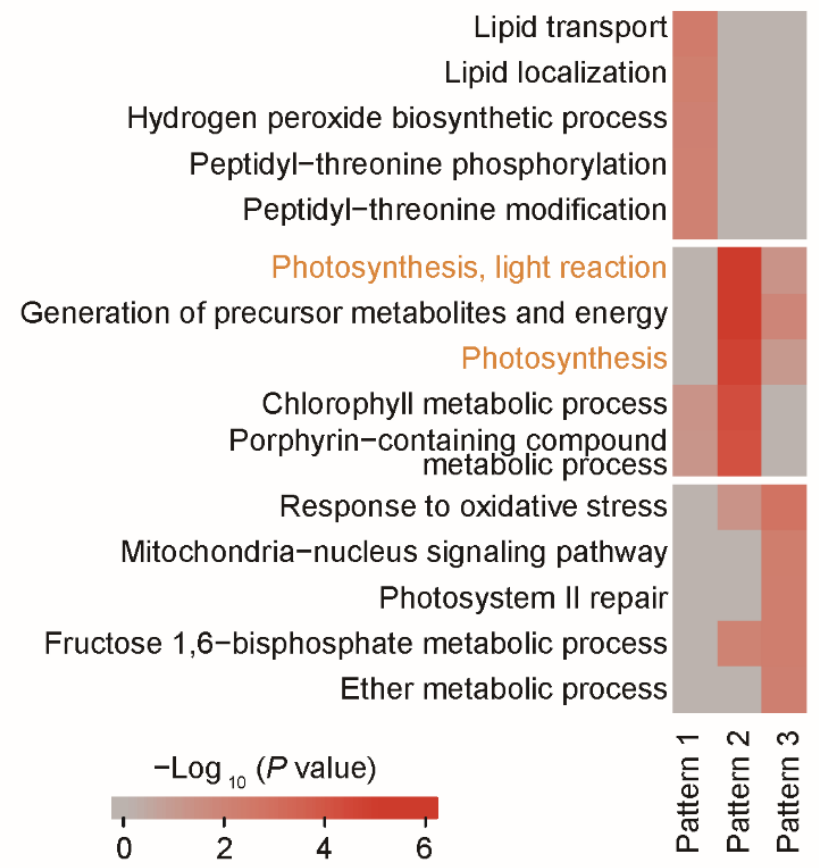

All cells

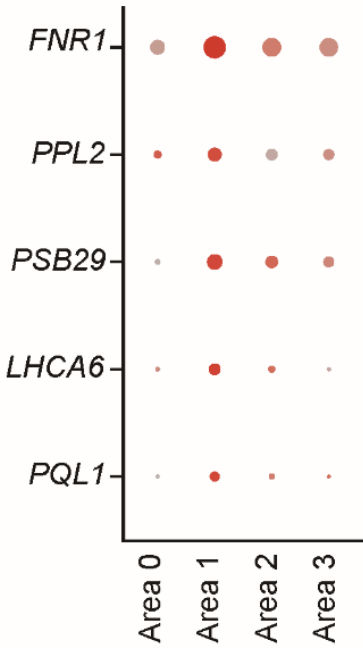

Percent expressed

$\begin{array}{cccc}\bullet & \bullet & \bullet & \bullet\end{array}$

Expression level

Low High

G

Mesophyll cells

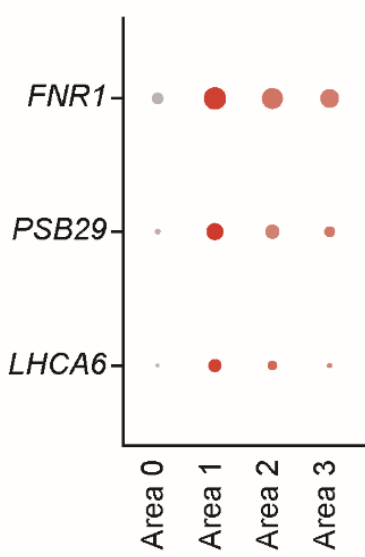

Percent expressed

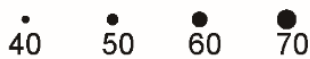

\section{Expression level}


Gopivprepindoi: atps://doi.org/10.1101/2021.10.20.465066; this version posted October 21, 2021. The copyright holder for this preprint 10 (n) certified by peer review) is the author/funder. All rights reserved. No reuse allowed without permission.

A

Vascular cells

Pseudotime
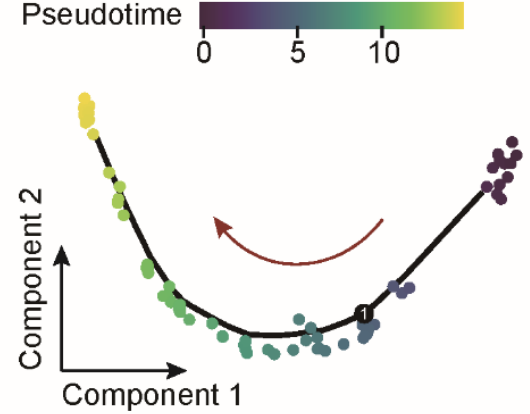

Area 0 - Area $1 \bullet$ Area $2 \bullet$ Area 3 Main vein .......- Leaf edge

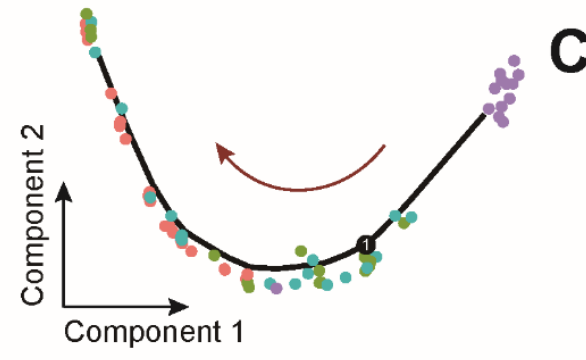

D - Guard cells - Epidermal cells

Pre-branch

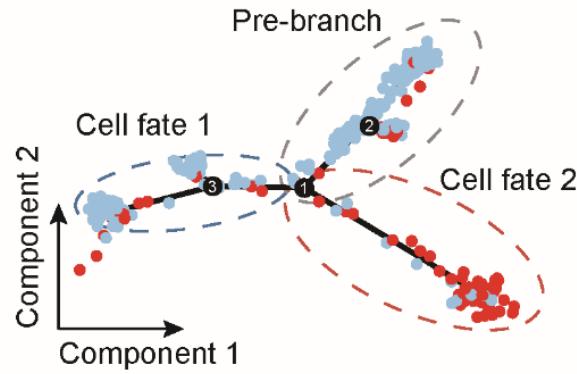

B

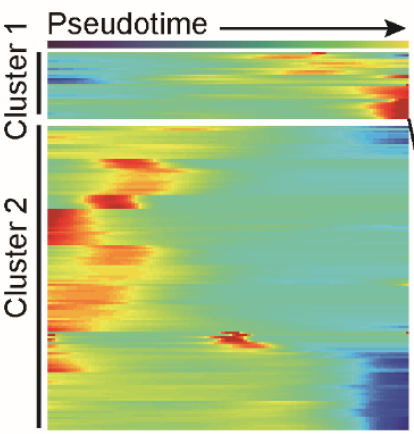

Toxin catabolic process $(P=1.81 \mathrm{E}-03)$

-Translational elongation $(P=1.97 \mathrm{E}-03)$

-Toxin metabolic process $(P=3.16 \mathrm{E}-03)$

-Cytoplasmic translation $(P=5.50 \mathrm{E}-03)$

Detoxification $(P=8.75 \mathrm{E}-03)$

-Photosynthesis $(P=1.19 \mathrm{E}-10)$

-Photosynthesis, light reaction $(P=3.55 \mathrm{E}-09)$

- Generation of precursor metabolites and energy $(P=1.56 \mathrm{E}-08)$

-Photosynthetic electron transport chain $(P=3.24 \mathrm{E}-07)$

-Electron transport chain $(P=1.94 \mathrm{E}-06)$

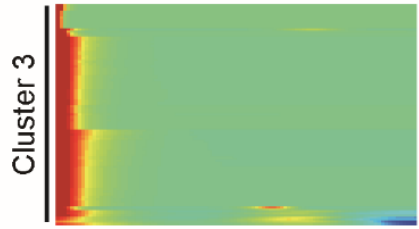

- Cellular response to hypoxia $(P=4.08 \mathrm{E}-04)$

- Cellular response to decreased oxygen levels $(P=4.27 \mathrm{E}-04)$

- Cellular response to oxygen levels $(P=4.27 \mathrm{E}-04)$

-Response to hypoxia $(P=7.16 \mathrm{E}-04)$

-Response to decreased oxygen levels $(P=7.74 \mathrm{E}-04)$

$$
\begin{array}{lllllll}
-3 & -2 & -1 & 0 & 1 & 2 & 3
\end{array}
$$

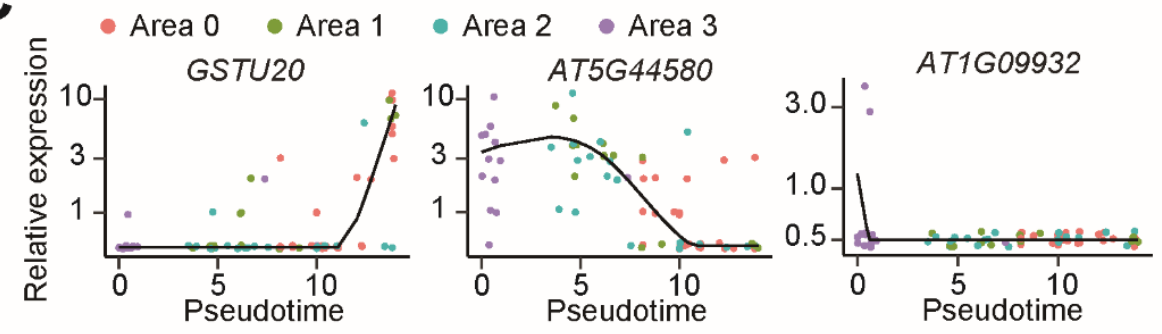

E

Pseudotime

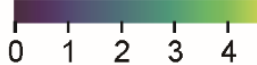

$\mathbf{F}$

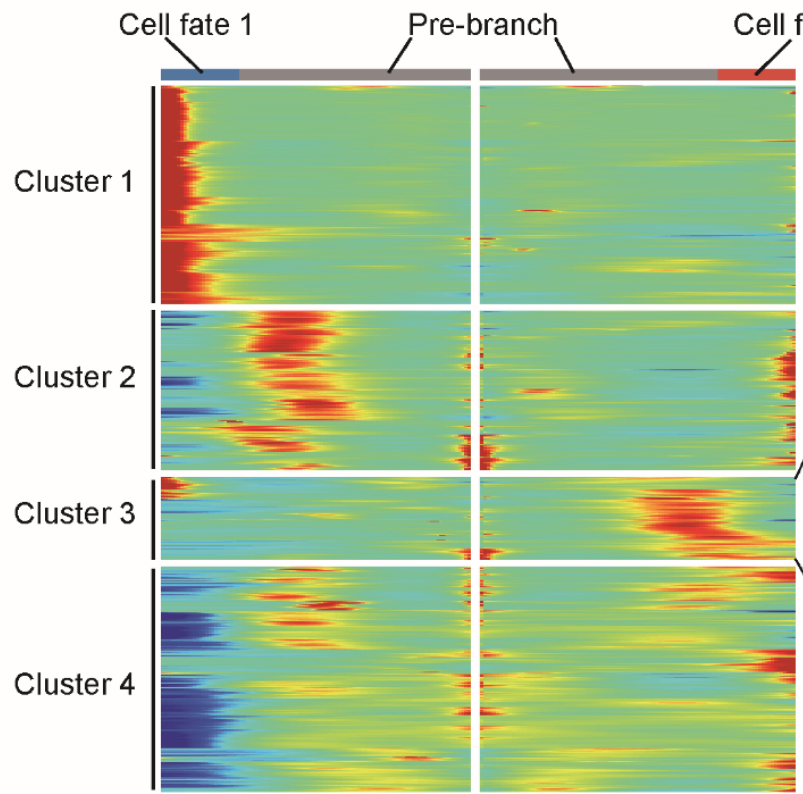

Cell fate 2

- Sulfur compound biosynthetic process $(P=6.30 \mathrm{E}-08)$

-Response to cadmium ion ( $P=4.62 \mathrm{E}-07)$

-Sulfur compound metabolic process $(P=9.31 \mathrm{E}-07)$

-Response to insect $(P=2.21 \mathrm{E}-06)$

-Response to metal ion ( $P=8.64 \mathrm{E}-06)$

-Cellular amino acid metabolic process $(P=4.77 \mathrm{E}-04)$

-Plastid organization $(P=7.15 \mathrm{E}-04)$

- Chloroplast organization $(P=1.01 \mathrm{E}-03)$

-AMP metabolic process $(P=1.43 \mathrm{E}-03)$

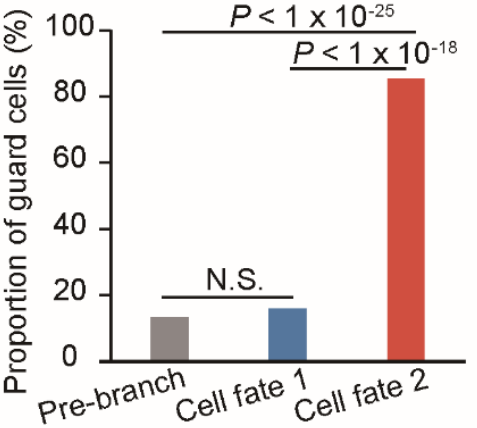

Translational elongation $(P=1.54 \mathrm{E}-3)$

- Cellular response to osmotic stress $(P=1.84 \mathrm{E}-03)$

-Photosynthesis $(P=6.99 \mathrm{E}-31)$

- Generation of precursor metabolites and energy $(P=5.30 \mathrm{E}-19)$

-Photosynthesis, light reaction $(P=4.74 \mathrm{E}-15)$

-Reductive pentose-phosphate cycle $(P=9.68 \mathrm{E}-14)$

-Photosynthesis, dark reaction $(P=2.03 \mathrm{E}-13)$ 


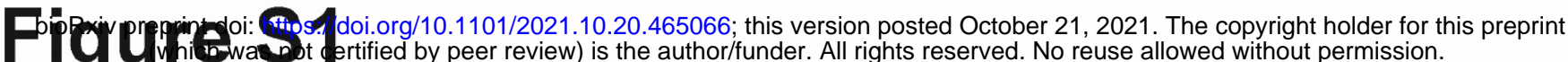
A

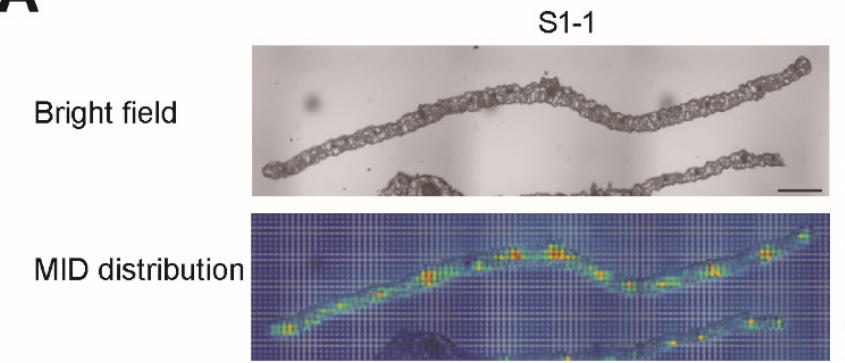

S1-5

Bright field

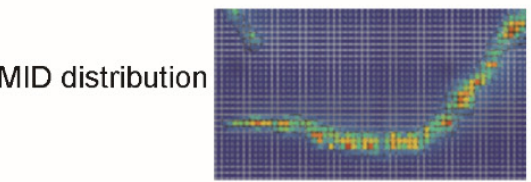

S1-7

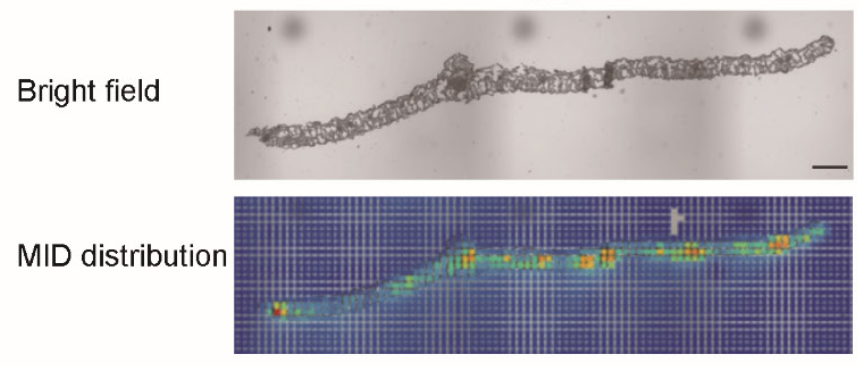

B
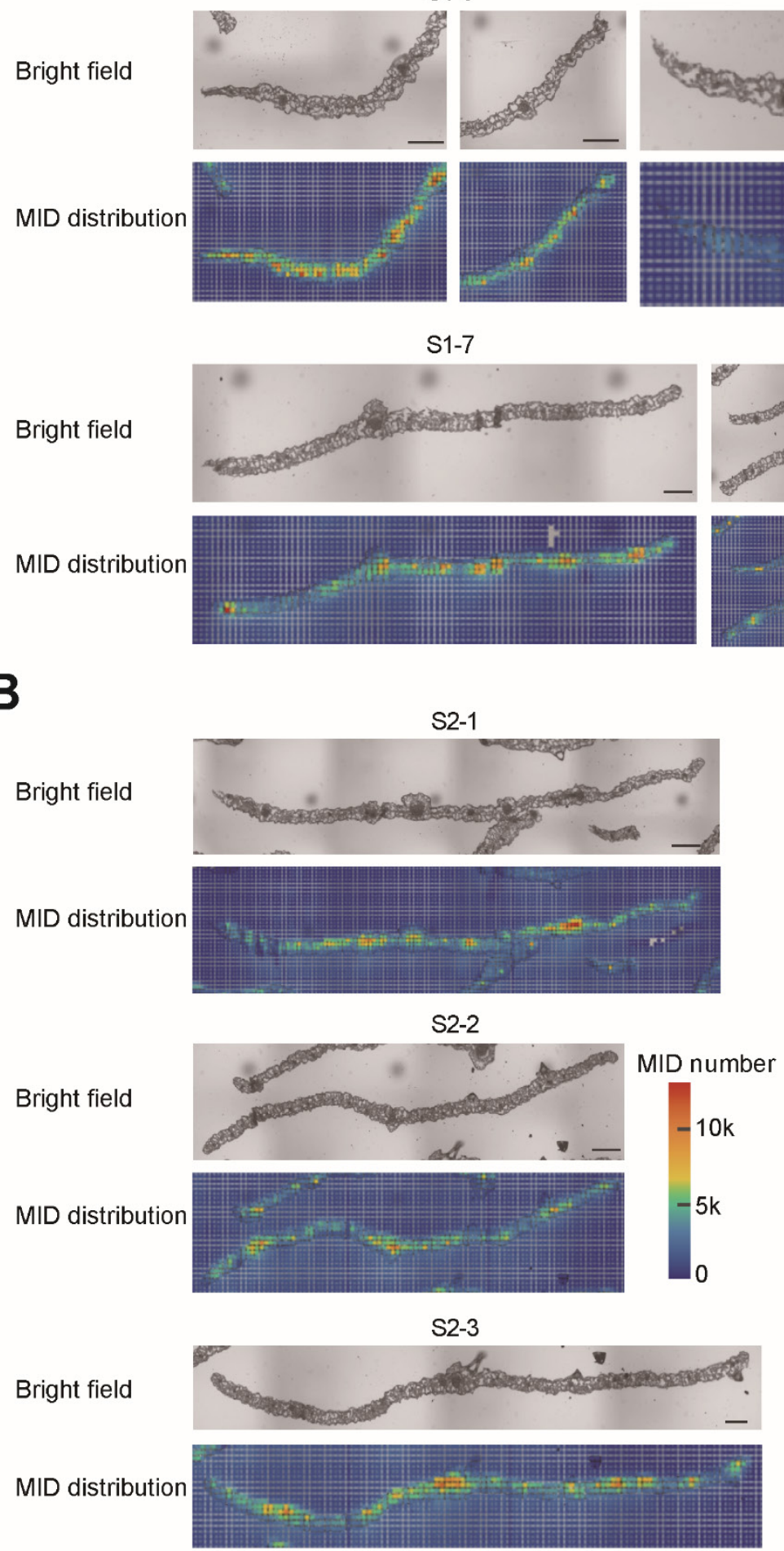

S1-3
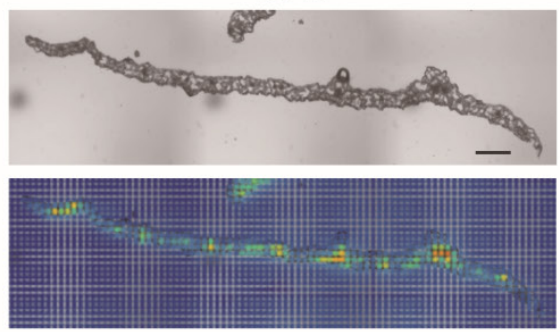

S1-6
S1-4
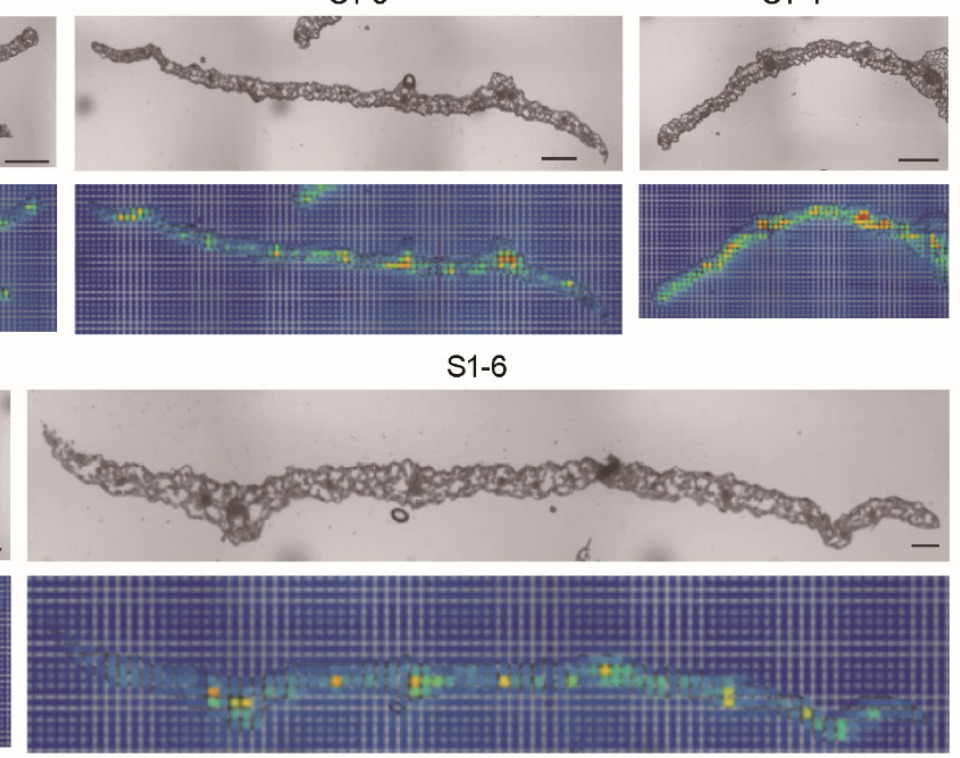

S1-8

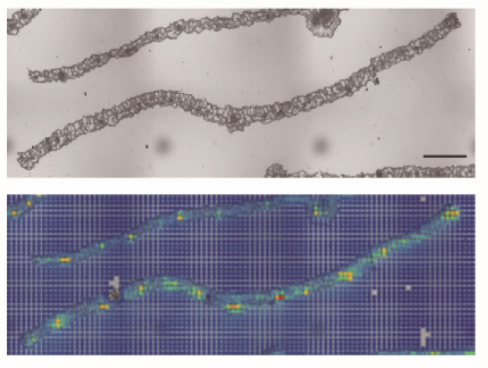

MID number

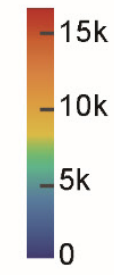

C

$$
\begin{aligned}
& \text { - } \mathrm{S} 1-1 \cdot \mathrm{S} 1-2 \text { - } \mathrm{S1-3} \cdot \mathrm{S} 1-4 \\
& \text { - } \mathrm{S} 1-5 \cdot \mathrm{S} 1-6 \text { - } \mathrm{S1-7} \cdot \mathrm{S1-8} \\
& \text { - } \mathrm{S} 2-1 \text { - } \mathrm{S} 2-2 \text { - } \mathrm{S} 2-3
\end{aligned}
$$

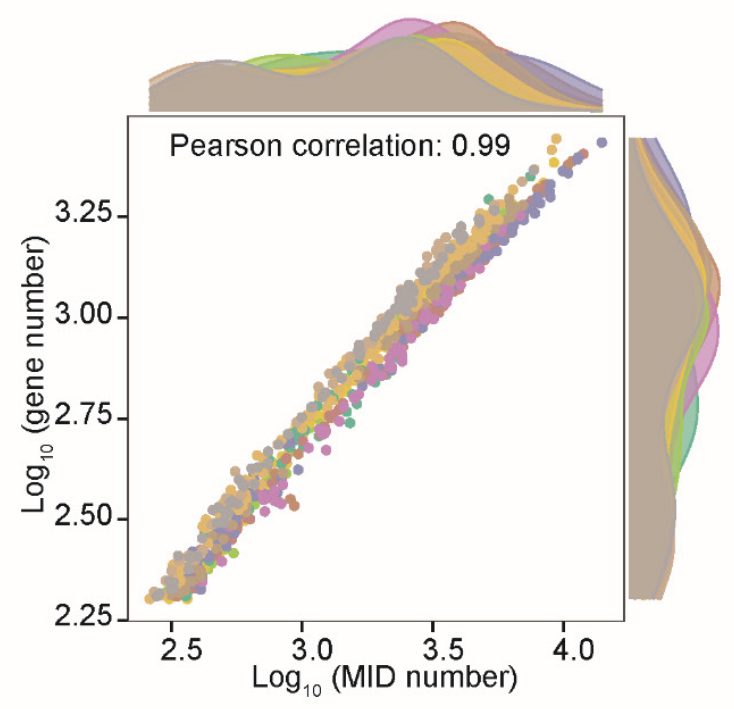




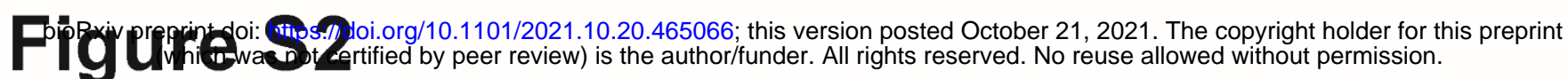

A

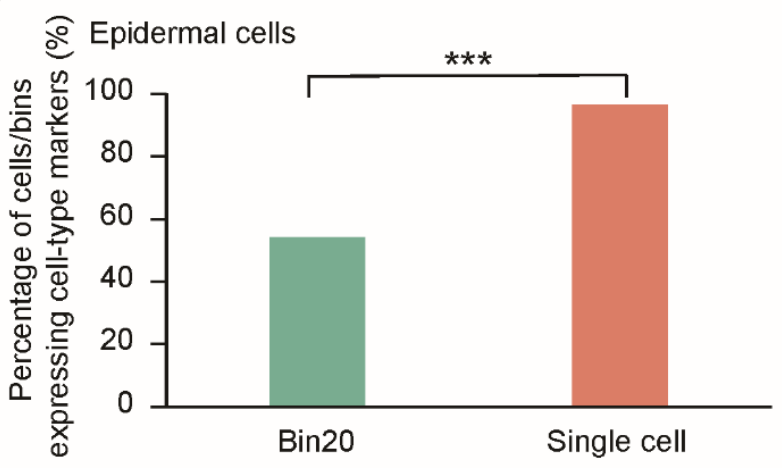

C

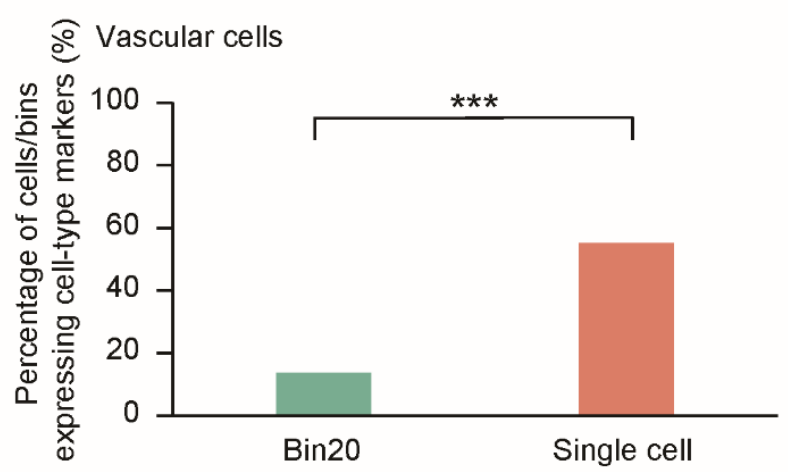

E

- Vascular cells $\quad$ Epidermal cells
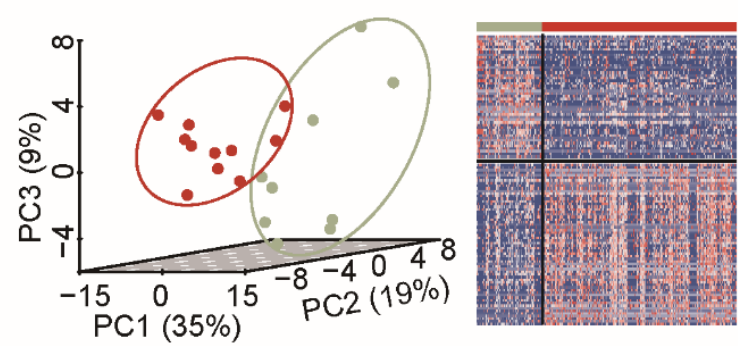

$G$
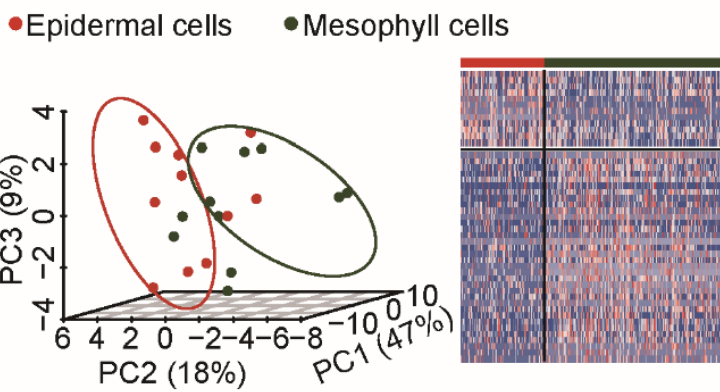

I

-Epidermal cells $\quad$ Guard cells
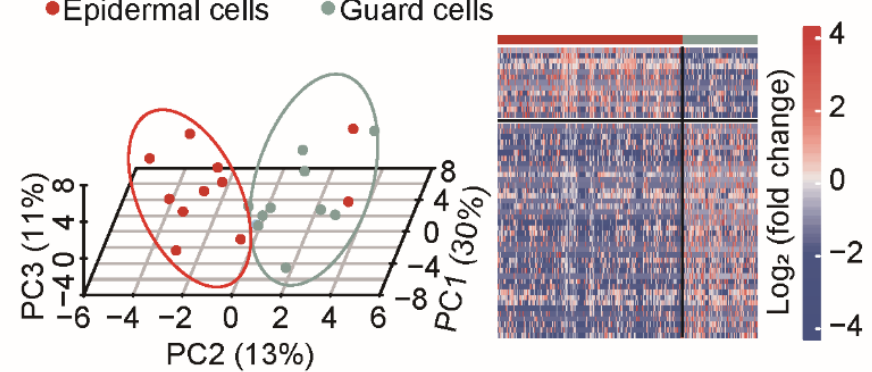

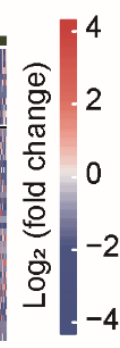

B

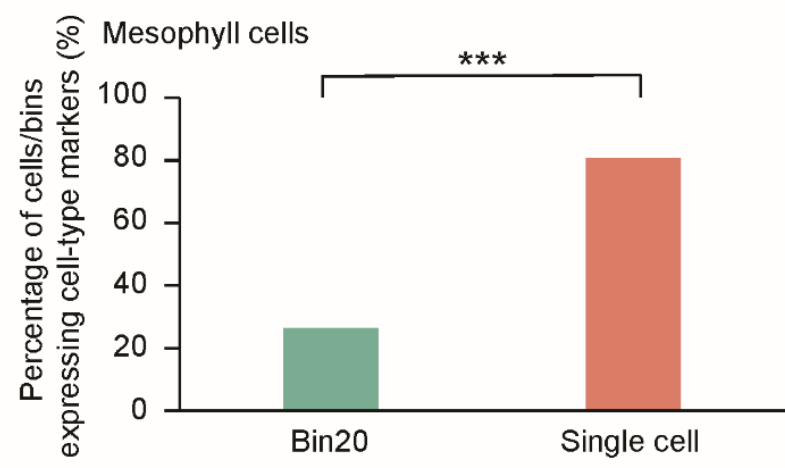

D

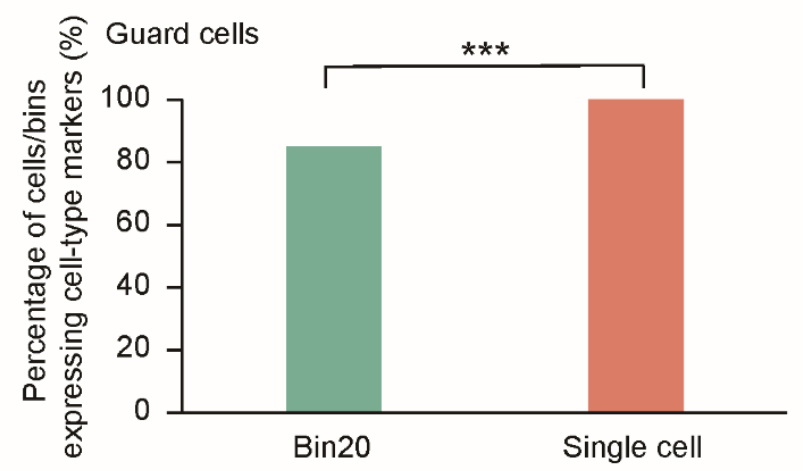

F - Vascular cells -Guard cells
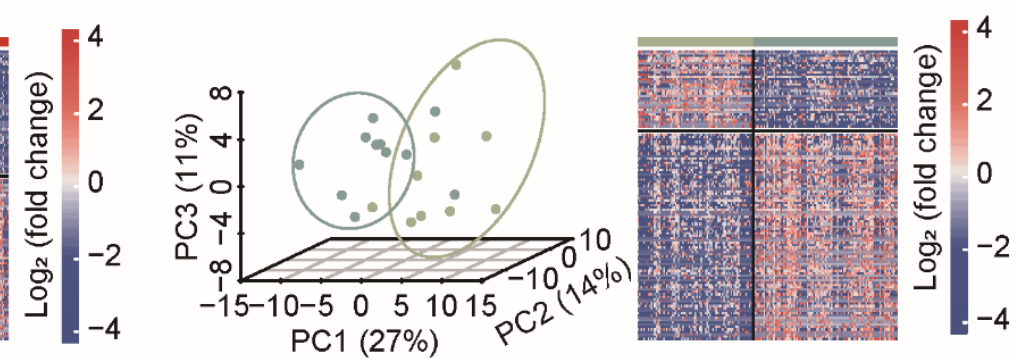

H

-Mesophyll cells • Guard cells
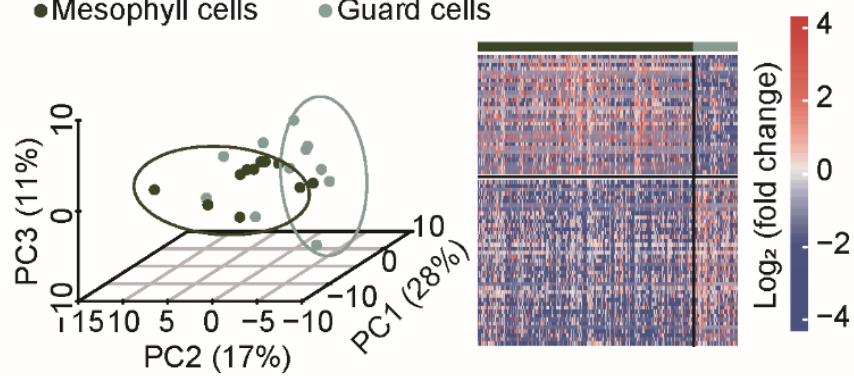
EDpexivprepringi: Atos?doi.org/10.1101/2021.10.20.465066; this version posted October 21, 2021. The copyright holder for this preprint

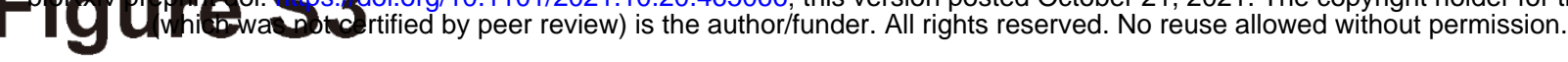

A
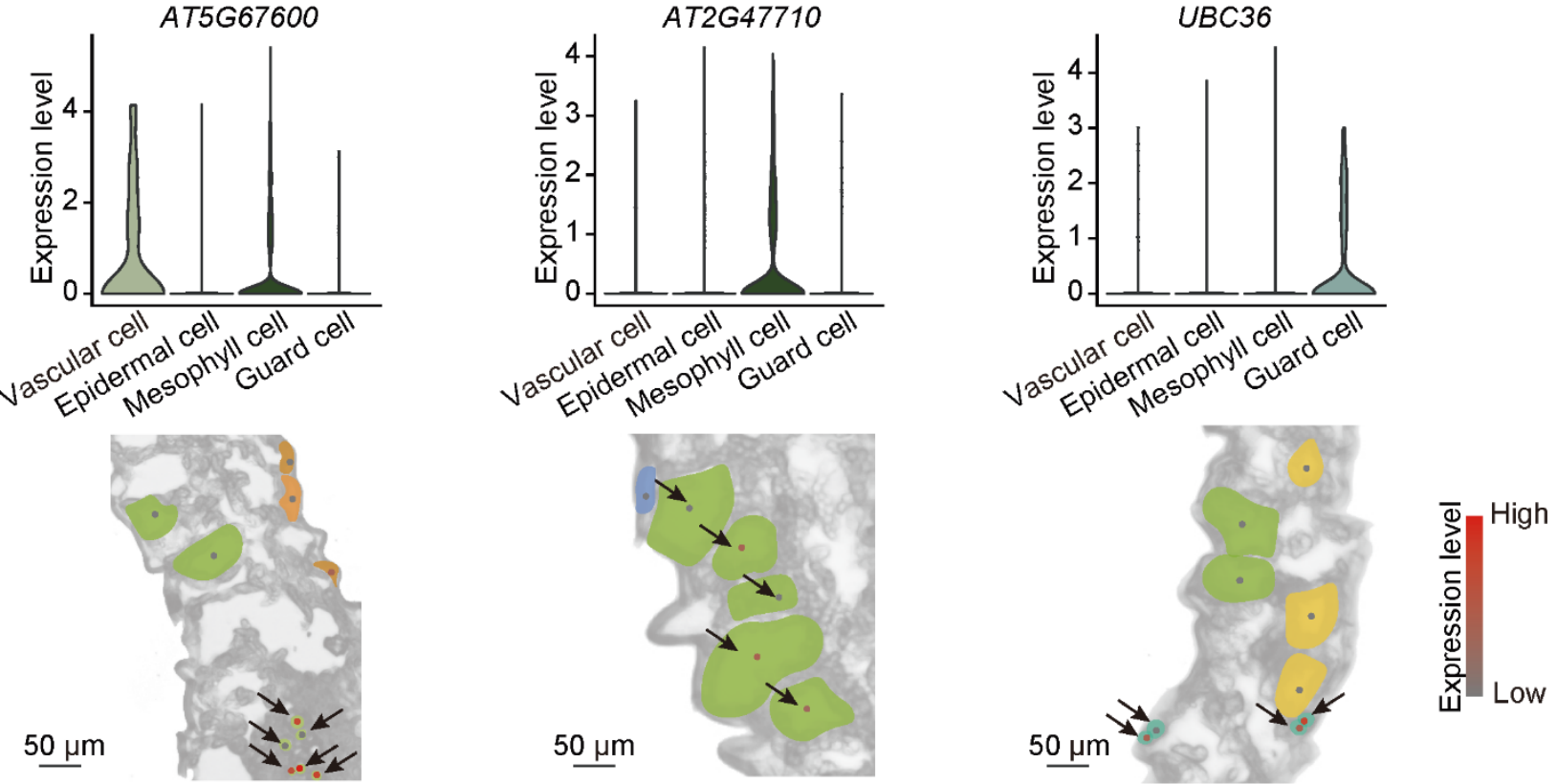

\begin{tabular}{l|l} 
- Upper epidermal cell & Epidermal cell
\end{tabular}

B

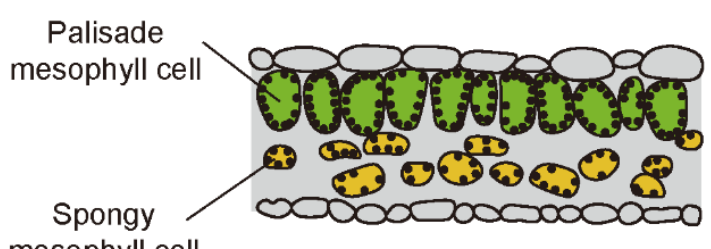

i) Single cell transcriptome alone

ii) Single cell transcriptome + spatial information
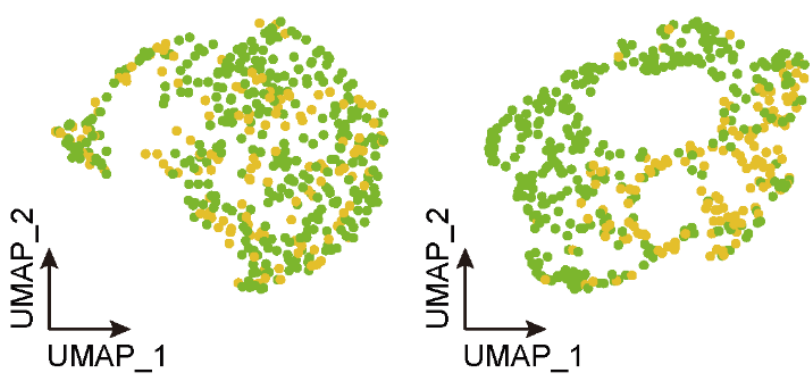

E
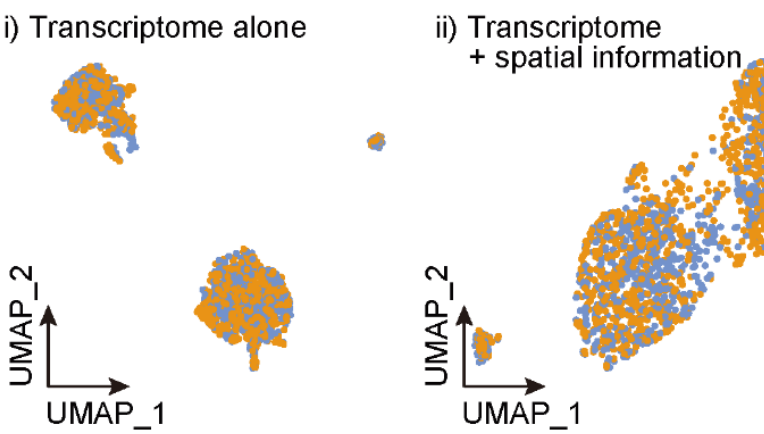

Bin from upper epidermal cell $\quad$ Bin from lower epidermal cell
C
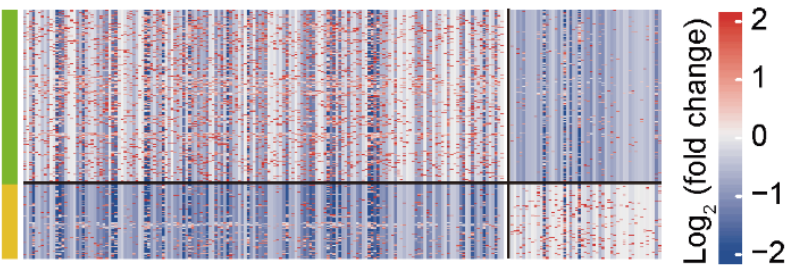

D

Palisade mesophyll cell @ Spongy mesophyll cell

Electron transport chain

Photosynthetic electron transport chain

Photosynthetic electron transport chain Photosynthesis ATP metabolic process Cellular response to hypoxia

Cellular response to oxygen levels Response to hypoxia

Response to oxygen levels Regulation of photosynthesis

Guard cell

Vascular cell 
Gopexivprepring oi: doi.org/10.1101/2021.10.20.465066; this version posted October 21, 2021. The copyright holder for this preprint

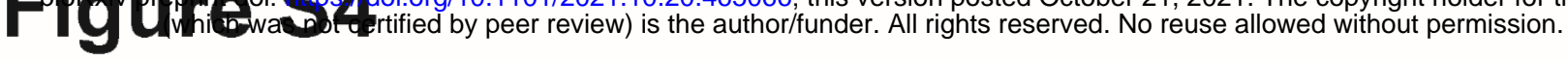

A

- Area 0 - Area 1 Area 2 Area 3

Main vein $-\ldots-\ldots$ Leaf edge

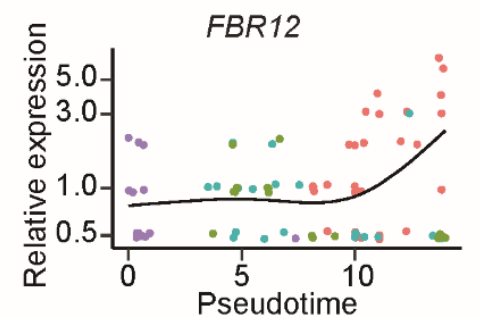

AT5G42530
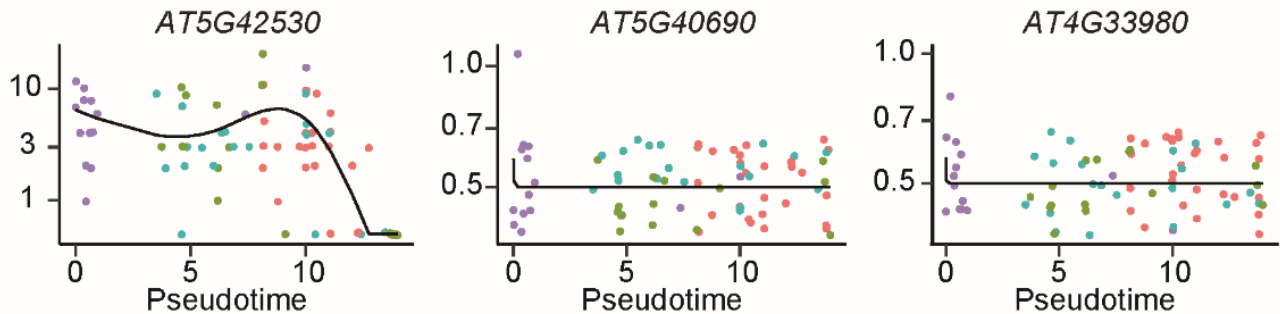

B

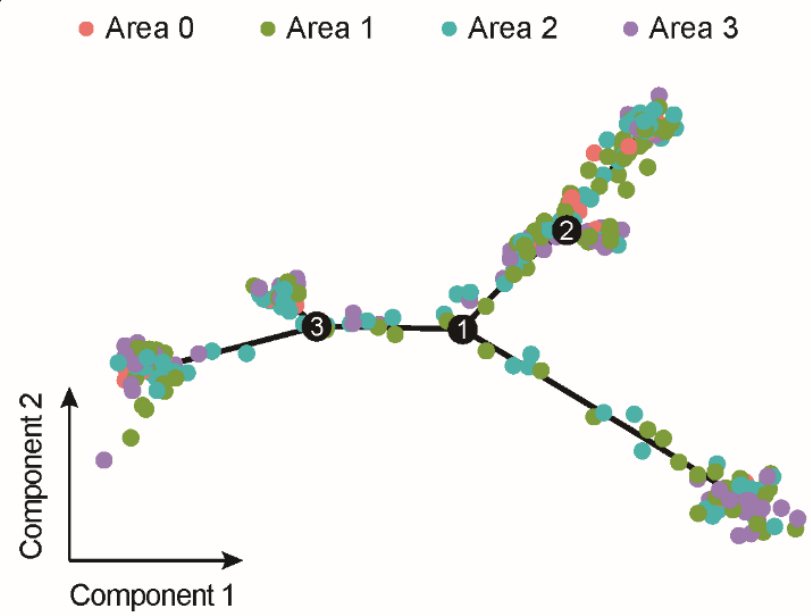

C

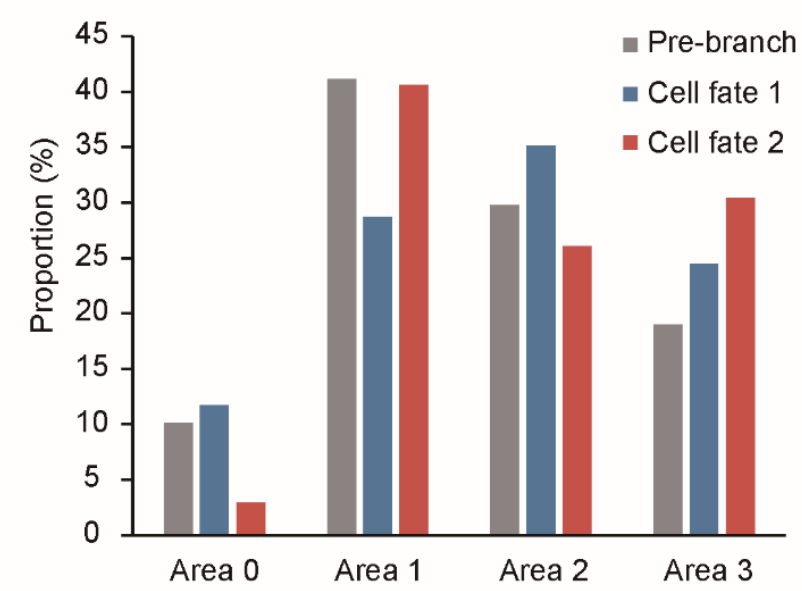

